\title{
Are inner disc misalignments common? ALMA reveals an isotropic outer disc inclination distribution for young dipper stars
}

\author{
M. Ansdell ${ }^{\oplus}, 1,2,3 \star$ E. Gaidos ${ }^{\oplus},{ }^{4}$ C. Hedges,${ }^{5}$ M. Tazzari ${ }^{\oplus},{ }^{6}$ A. L. Kraus, ${ }^{7}$ \\ M. C. Wyatt ${ }^{\oplus}, 6$ G. M. Kennedy ${ }^{\oplus}, 8,9$ J. P. Williams, ${ }^{10}$ A. W. Mann, ${ }^{11}$ I. Angelo, ${ }^{2,12}$ \\ G. Dûchene ${ }^{\oplus},{ }^{2,13}$ E. E. Mamajek, ${ }^{14,15}$ J. Carpenter, ${ }^{16}$ T. L. Esplin ${ }^{17}$ and A. C. Rizzuto ${ }^{7}$ \\ ${ }^{1}$ Center for Integrative Planetary Science, University of California at Berkeley, Berkeley, CA 94720, USA \\ ${ }^{2}$ Department of Astronomy, University of California at Berkeley, Berkeley, CA 94720, USA \\ ${ }^{3}$ Flatiron Institute, Simons Foundation, 162 Fifth Ave, New York, NY 10010, USA \\ ${ }^{4}$ Department of Earth Sciences, University of Hawai' $i$ at Mānoa, Honolulu, HI 96822, USA \\ ${ }^{5}$ NASA Ames Research Center, Moffett Blvd, Mountain View, CA 94035, USA \\ ${ }^{6}$ Institute of Astronomy, University of Cambridge, Madingley Road, Cambridge CB3 OHA, UK \\ ${ }^{7}$ Department of Astronomy, The University of Texas at Austin, Austin, TX 78712, USA \\ ${ }^{8}$ Department of Physics, University of Warwick, Gibbet Hill Road, Coventry CV4 7AL, UK \\ ${ }^{9}$ Centre for Exoplanets and Habitability, University of Warwick, Gibbet Hill Road, Coventry CV4 7AL, UK \\ ${ }^{10}$ Institute for Astronomy, University of Hawai 'i at Mānoa, Honolulu, HI 96822, USA \\ ${ }^{11}$ Department of Physics and Astronomy, University of North Carolina at Chapel Hill, Chapel Hill, NC 27599, USA \\ ${ }^{12}$ Department of Physics and Astronomy, University of California, Los Angeles, Los Angeles, CA 90095, USA \\ ${ }^{13}$ CNRS Institut de Planétologie et d'Astrophysique (IPAG), Université Grenoble-Alpes, F-38000 Grenoble, France \\ ${ }^{14}$ Jet Propulsion Laboratory, California Institute of Technology, 4800 Oak Grove Dr., Pasadena, CA 91109, USA \\ ${ }^{15}$ Department of Physics and Astronomy, University of Rochester, Rochester, NY 14627, USA \\ ${ }^{16}$ Joint ALMA Observatory, Avenida Alonso de Córdova 3107, JC22+54 Vitacura, Santiago, Chile \\ ${ }^{17}$ Steward Observatory, University of Arizona, Tucson, AZ 85719, USA
}

Accepted 2019 November 28. Received 2019 November 28; in original form 2019 September 27

\begin{abstract}
Dippers are a common class of young variable star exhibiting day-long dimmings with depths of up to several tens of per cent. A standard explanation is that dippers host nearly edge-on $\left(i_{\mathrm{d}} \approx 70^{\circ}\right)$ protoplanetary discs that allow close-in $(<1 \mathrm{au})$ dust lifted slightly out of the mid-plane to partially occult the star. The identification of a face-on dipper disc and growing evidence of inner disc misalignments brings this scenario into question. Thus, we uniformly (re)derive the inclinations of 24 dipper discs resolved with (sub-)mm interferometry from ALMA. We find that dipper disc inclinations are consistent with an isotropic distribution over $i_{\mathrm{d}} \approx 0-75^{\circ}$, above which the occurrence rate declines (likely an observational selection effect due to optically thick disc mid-planes blocking their host stars). These findings indicate that the dipper phenomenon is unrelated to the outer ( $>10 \mathrm{au}$ ) disc resolved by ALMA and that inner disc misalignments may be common during the protoplanetary phase. More than one mechanism may contribute to the dipper phenomenon, including accretion-driven warps and 'broken' discs caused by inclined (sub-)stellar or planetary companions.
\end{abstract}

Key words: planet-star interactions-protoplanetary discs-binaries: visual-stars: variables: T Tauri, Herbig Ae/Be.

\section{INTRODUCTION}

Photometric variability is a hallmark of young $(\lesssim 10 \mathrm{Myr})$ stars and studies of this variability provide insight into the physical processes underpinning early stellar evolution and planet formation. Some young stars transiently dim due to obscuration by circumstellar dust,

${ }^{\star}$ E-mail: megan.ansdell@gmail.com a phenomenon first noted in ground-based photometry of bright intermediate-mass Herbig Ae/Be stars that fade up to several orders of magnitude for weeks to months, named UXOR variables after the archetype UX Orionis (Herbst et al. 1994). More sensitive spacebased telescopes - most notably CoRoT (Baglin et al. 2006), Spitzer (Fazio et al. 2004), and Kepler (Borucki et al. 2010) - later identified a related class of fainter, typically late-type pre-main sequence stars known as 'dippers' (Alencar et al. 2010; Morales-Calderón et al. 2011; Cody et al. 2014; Ansdell et al. 2016b; Stauffer et al. 2017). 
The dippers exhibit more moderate dimming events, with depths up to several tens of percent and durations of roughly $1 \mathrm{~d}$, and can be either quasi-periodic or episodic (e.g. Cody et al. 2014) with diverse profile shapes (e.g. see fig. 4 in Ansdell et al. 2016b). Dippers are particularly interesting for studying disc evolution and planet formation as they are common, making up 20-30 per cent of young stellar populations (Alencar et al. 2010; Cody et al. 2014).

The dipper phenomenon is thought to result from partial occultations of the star by circumstellar dust. The dust interpretation is supported by dippers invariably having infrared emission in excess of that expected from the stellar photosphere, indicating the presence of a protoplanetary disc, as well as the dips often being shallower at longer wavelengths, where dust is less scattering (Morales-Calderón et al. 2011; Cody et al. 2014; Schneider et al. 2018). An origin in the inner $(<1 \mathrm{au})$ disc is suggested by the quasi-periodic dippers having periods of a few days, which is often indistinguishable from the stellar rotation period (Bodman et al. 2017), as well as a positive correlation between dip depth and excess emission in the WISE $4.6 \mu \mathrm{m}$ band, which is sensitive to warm dust grains near the disc-star co-rotation radius around late-type stars (Ansdell et al. 2016b).

The physical mechanism identified early on to explain the prototypical dipper AA Tau invokes magnetospheric accretion to lift dusty material out of the disc mid-plane, creating an inner warp that occults the star (Bouvier et al. 1999). A prediction of this model is that discs around dipper stars should be observed at nearly edgeon $\left(i_{\mathrm{d}} \approx 70^{\circ}\right)$ inclinations, since lower inclinations would preclude the occultation while higher inclinations would cause the optically thick disc mid-plane to obscure the star entirely. Indeed, McGinnis et al. (2015) and Kesseli et al. (2016) reproduced the light curves of dippers in NGC 2264 obtained by Spitzer and CoRoT using models of magnetospheric accretion from nearly edge-on protoplanetary discs. Bodman et al. (2017) later revised this accretion warp model with magnetospheric truncation theory to show that it could explain dippers with discs of only moderate inclinations down to $i_{\mathrm{d}} \approx 50^{\circ}$.

The expectation of dipper systems tending to have high inclinations can be tested by resolving the discs with infrared or (sub)mm interferometry. Although the infrared probes closer to the star $(<1 \mathrm{au})$ where the dipper phenomenon likely originates, the faintness of the dippers often prohibits these observations. This is not the case for (sub-)mm interferometry, which with the advent of the Atacama Large sub-millimeter/Millimeter Array (ALMA) can now quickly resolve protoplanetary discs around all stellar types, but is sensitive to the outer ( $>10 \mathrm{au}$ ) disc. In our previous work (Ansdell et al. 2016a), we used archival ALMA data of three dippers to show that their outer discs ranged from face-on to edge-on. This hinted towards significantly misaligned inner disc components and/or the need for other dipper mechanisms. Evidence of misaligned inner discs has also been recently inferred for several systems using highcontrast optical/infrared images, which have revealed shadows in the outer disc cast by unseen inclined inner disc components (e.g. Marino, Perez \& Casassus 2015; Stolker et al. 2016; Debes et al. 2017; Benisty et al. 2018; Casassus et al. 2018). A notable example is the dipper J1604, which hosts a face-on transition disc resolved by ALMA (Ansdell et al. 2016a) and variable shadows seen by VLT/SPHERE (Pinilla et al. 2018a), suggesting a highly misaligned $\left(\sim 70-90^{\circ}\right)$ and dynamic inner disc component.

In this work, we uniformly (re)analyse resolved (sub-)mm ALMA data for two dozen dipper discs in an effort to robustly infer the distribution of their outer disc inclinations. In Section 2, we present our sample and describe the data sets used in this work. We derive disc inclinations from the ALMA data in Section 3, then in Section 4 we construct the dipper disc inclination distribution and discuss its impact on our understanding of the dippers and inner disc misalignments. We conclude in Section 5 and suggest avenues for future work.

\section{SAMPLE AND DATA SETS}

\subsection{Sample}

Our sample consists of all known dippers in the $\rho$ Ophiuchi ( $\rho$ Oph) and Upper Scorpius (Upper Sco) star-forming regions that have been identified by their $K 2$ Campaign $2(K 2 / \mathrm{C} 2)$ light curves and have discs resolved by ALMA. We focus on these two nearby $(\approx 130 \mathrm{pc}$; Gagné et al. 2018) star-forming regions because they are known to host numerous dippers (e.g. Cody \& Hillenbrand 2018) and have been surveyed extensively with ALMA (e.g. Barenfeld et al. 2016; Cieza et al. 2018). Moreover, they were observed during the same $K 2$ campaign and thus contain the same systematics in their light curves (see Section 2.2).

The dippers in our sample were all previously identified based on their K2/C2 light curves by Ansdell et al. (2016b), Hedges, Hodgkin \& Kennedy (2018), and/or Cody \& Hillenbrand (2018) using different methods. Ansdell et al. (2016b) worked with citizen scientists to identify 25 dippers by eye and study 10 of them in detail. Hedges et al. (2018) then used these 25 dippers to train a supervised machine learning algorithm with a random forest classifier to expand the sample to 95 dippers. Cody \& Hillenbrand (2018) employed their traditional statistics of periodicity and symmetry to categorize variable young stars in the K2/C2 data set, identifying 94 dippers. Combining these samples results in 122 unique dippers.

The issue with combining these samples is that significantly different criteria were used to identify the dippers, and sometimes the methods did not all agree. Therefore, we consider all the dippers in the combined samples, then require at least three dips to be present in the $K 2 / \mathrm{C} 2$ light curves and apply the $R_{\text {dip }}>5.0$ selection criteria of Ansdell et al. (2016b). To summarize the more detailed description of $R_{\text {dip }}$ in Ansdell et al. (2016b): $R_{\text {dip }}$ is the ratio of the average depth of the three deepest dips to the standard deviation for a normalized light curve that has been put through a highpass filter with a cut-on frequency of $1 \mathrm{~d}^{-1}$ (the high-pass filter preserves the dips while suppressing the periodic variability from stellar rotation due to the different duty cycles). This criteria helps to avoid stars with large intrinsic variability (e.g. stochastic variables) as well as noisy light curves with suspicious irregularities related to instrumental effects (e.g. charge bleed) or data corruption (e.g. data discontinuities). This cut reduces the sample to 79 dippers.

We then use data from our targeted ALMA programme as well as a search of the ALMA archive to identify those with resolved circumstellar discs (see Section 2.3 for a description of the ALMA data sets used in this work, Section 3.1 for the derivation of the disc inclinations, and Appendix $\mathrm{C}$ for a discussion of the dippers observed but not resolved by ALMA). We note that a general literature search did not return any dippers with disc inclinations derived only from pre-ALMA radio interferometers, and only one known dipper has an inclination derived only from scattered light (EPIC 204206295 or DoAr 28 with a modestly inclined disc of $i_{\mathrm{d}}=50_{-10}^{+15}$; Rich et al. 2015), which we do not include in our sample. Our final sample therefore consists of 24 dippers, 12 in $\rho$ Oph and 12 in Upper Sco. Their basic properties are presented in Table 1 and their ALMA data and K2/C2 light curves are shown in Fig. 1. 


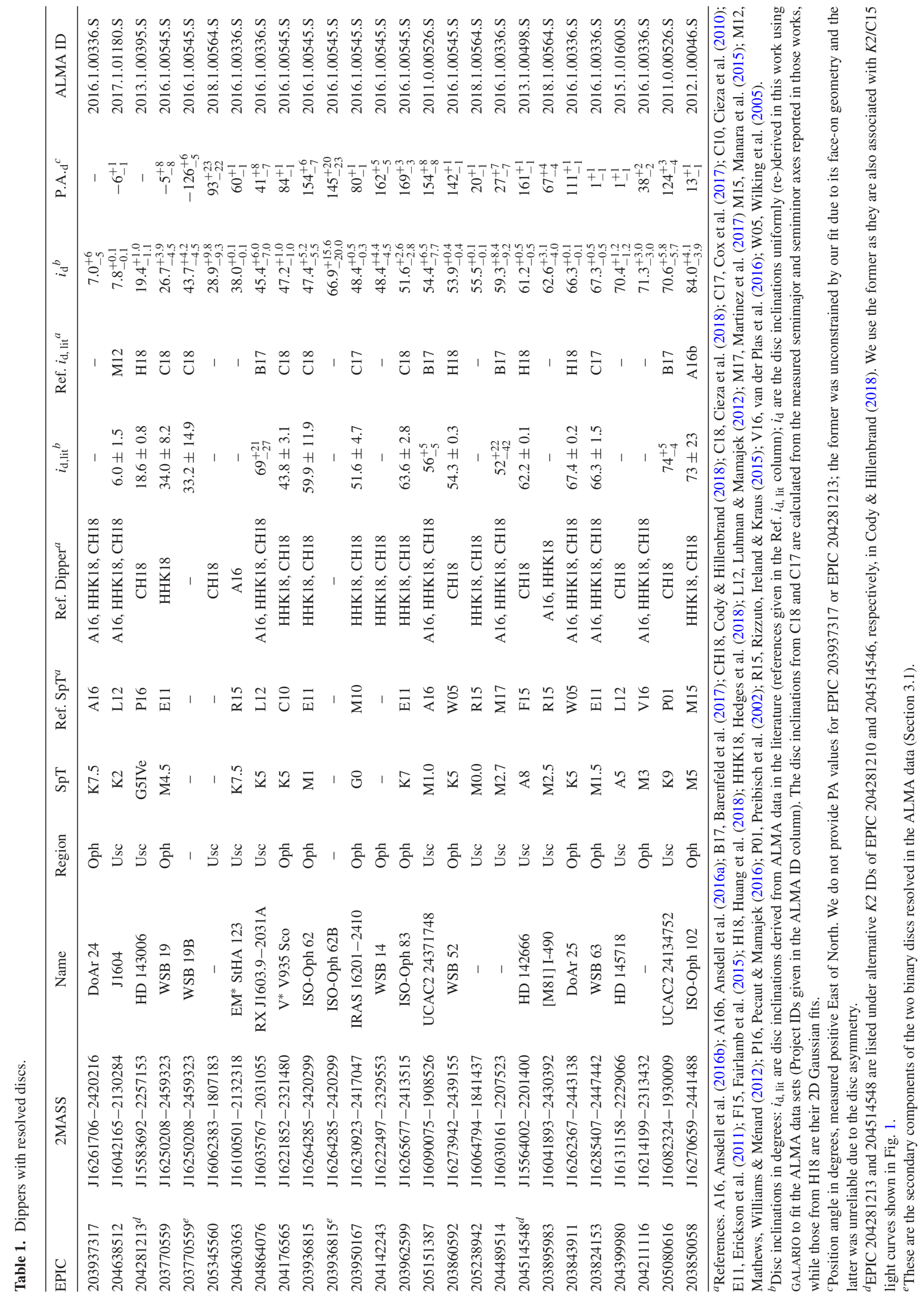



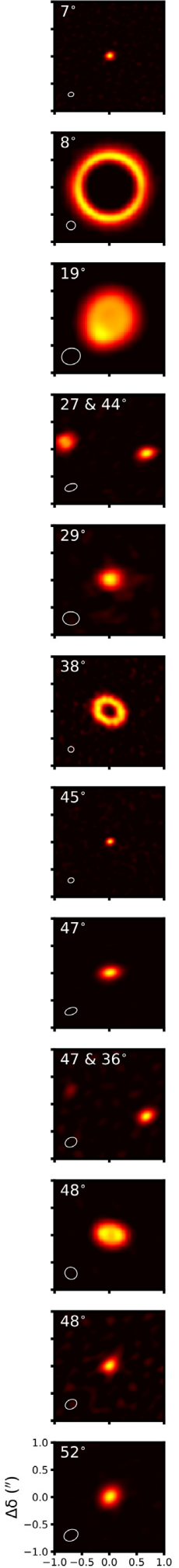

$\Delta \alpha(")$
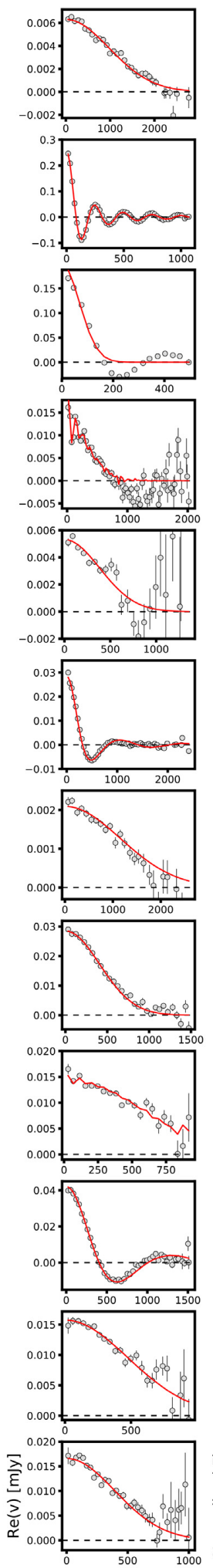

UV Distance $[k \lambda]$
悉

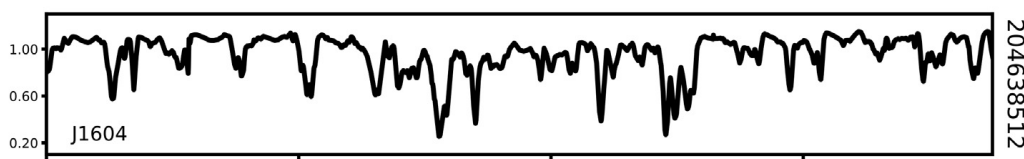
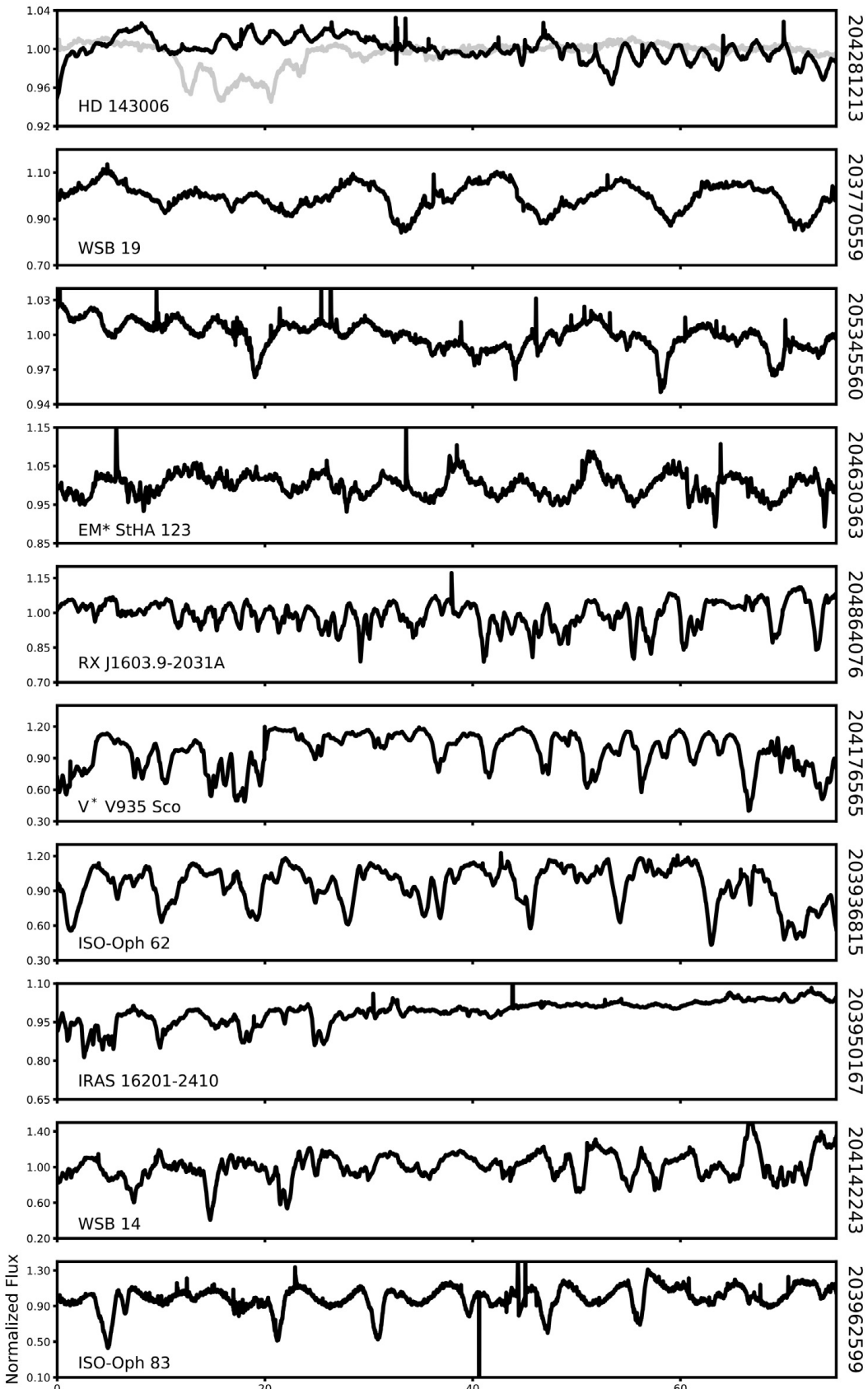

Time (days)

Figure 1. ALMA images (left) and associated visibilities (middle) as well as $K 2 / \mathrm{C} 2$ light curves (right) for the sources in our sample. The ALMA images are $2 \operatorname{arcsec} \times 2 \operatorname{arcsec}($ corresponding to about $260 \times 260 \mathrm{au}$ ) with the beam shown by the white ellipse and the disc inclination given for reference. The GALARIO model fits are shown by the red line over the visibility data, which are de-projected using the best-fitting GALARIO geometries; declining visibilities with UV distance indicate resolved sources (for the binaries we show the combined visibilities). The EPIC names of each source are on the far right, and common names are given in the K2/C2 panels (see Table 1). The K2/C15 light curves for EPIC 204281213, 204489514, and 204514548 are overlaid in gray over their K2/C2 data. 

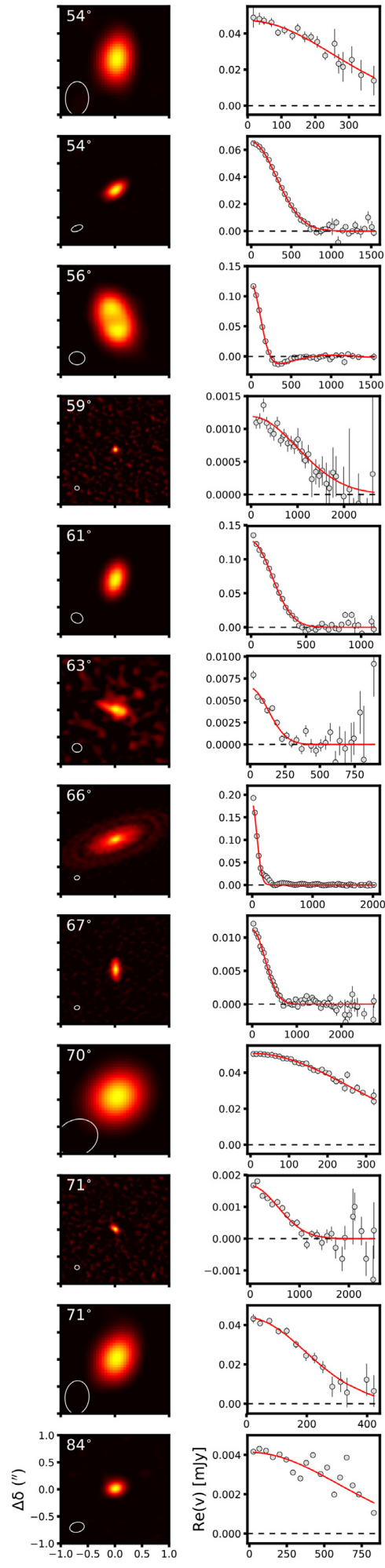

$\Delta \alpha\left(^{\prime \prime}\right)$
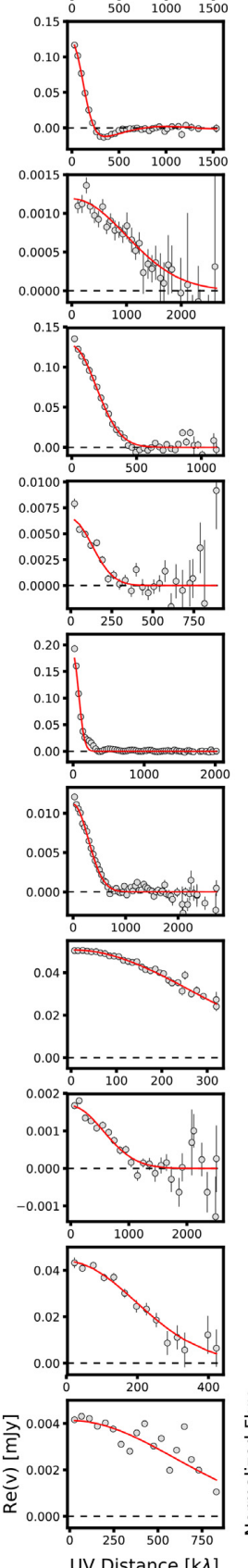
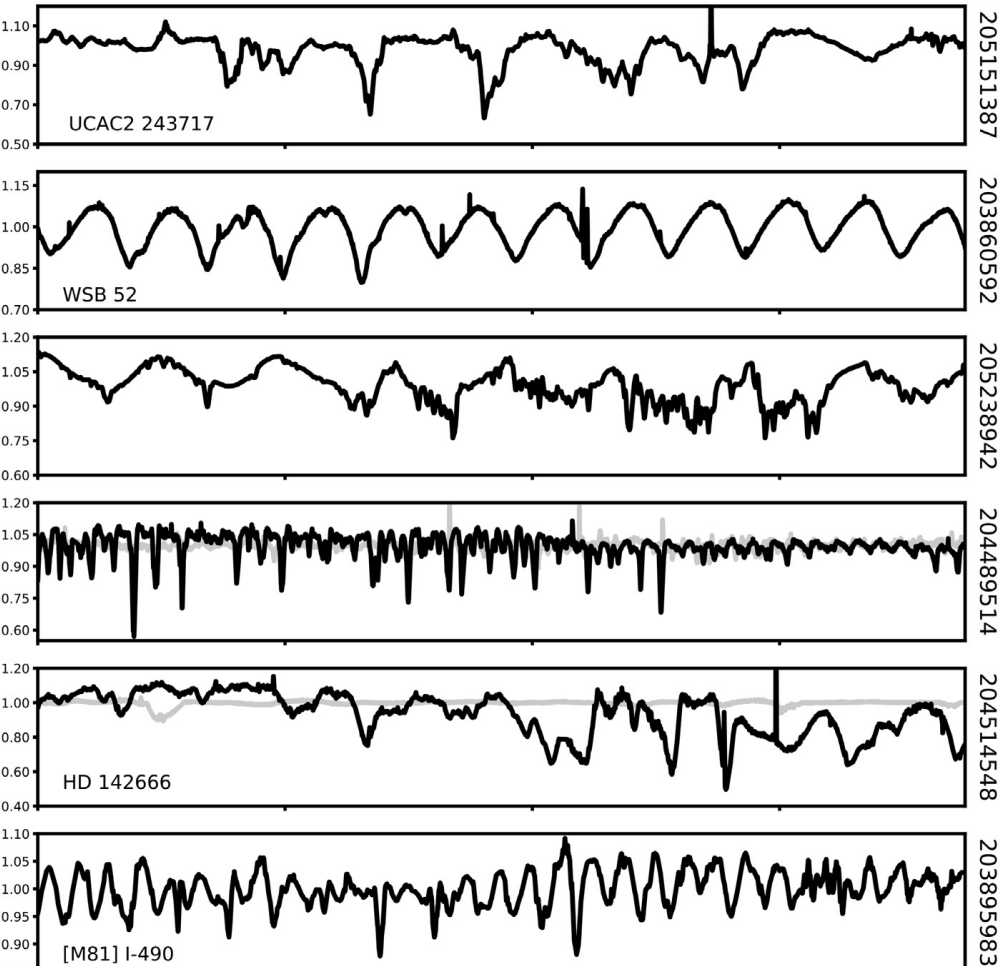

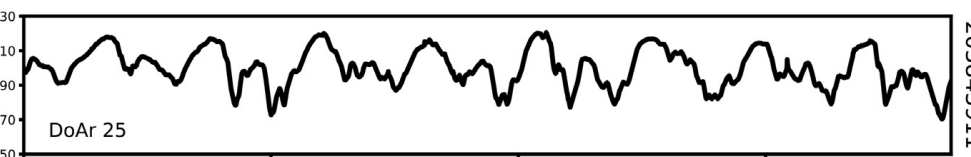
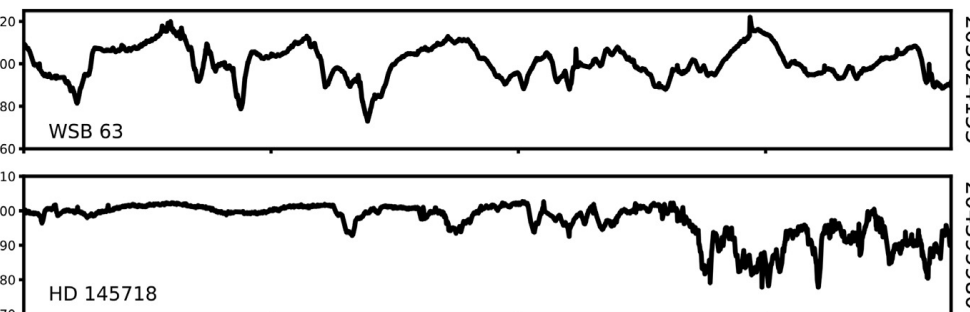

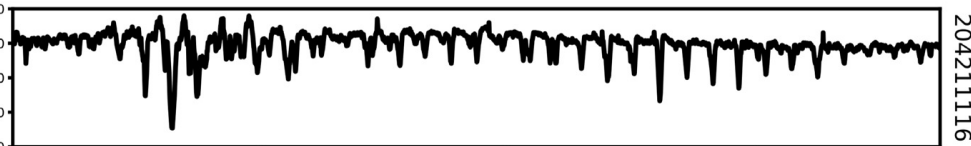

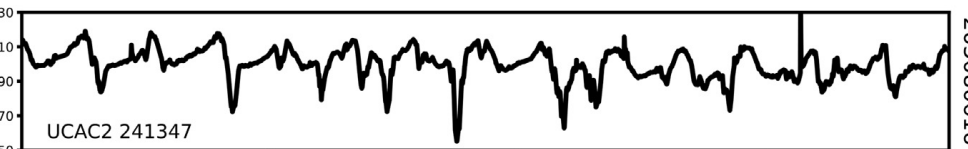

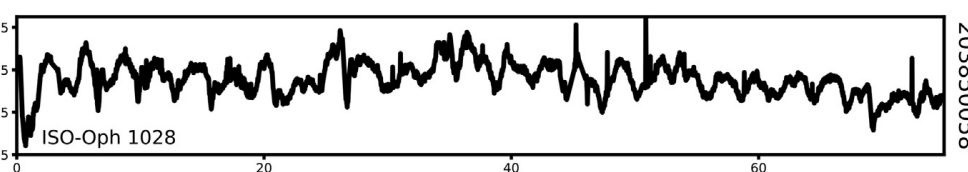

Figure 1 - continued

\section{$2.2 K 2$ light curves}

To construct the $K 2 / \mathrm{C} 2$ light curves used in this work, we reextract the photometry for each dipper from the original pixellevel data. This is needed because $K 2$ (Howell et al. 2014), the successor mission to Kepler after the spacecraft lost two out of its four reaction wheels, adopted an ecliptic-observing orientation to stabilize its pointing using solar radiation pressure as a pseudo third reaction wheel. Due to Sun-angle constraints, $K 2$ observations 
Table 2. Candidate companions detected in AO images.

\begin{tabular}{|c|c|c|c|c|c|c|}
\hline EPIC & $\begin{array}{c}\rho \\
\text { (mas) }\end{array}$ & $\begin{array}{c}\text { PA } \\
(\mathrm{deg})\end{array}$ & $\begin{array}{c}\Delta m \\
(\mathrm{mag})\end{array}$ & Band & $\begin{array}{l}\text { Epoch }^{a} \\
\text { (MJD) }\end{array}$ & Ref. $^{b}$ \\
\hline 203843911 & $3697.3 \pm 1.8$ & $357.308 \pm 0.027$ & $8.737 \pm 0.112$ & $K_{\mathrm{p}}+\mathrm{C} 600$ & 56116.35 & TW \\
\hline 203895983 & $296.23 \pm 1.53$ & $70.203 \pm 0.290$ & $0.109 \pm 0.006$ & $K_{\mathrm{p}}$ & 57195.38 & TW \\
\hline 203936815 & $1438 \pm 12$ & $69.5 \pm 0.3$ & $1.311 \pm 0.022$ & $K_{\mathrm{S}}$ & 51713 & R05 \\
\hline 203950167 & $1900 \pm 100$ & $38.4 \pm 1.0$ & 2.70 & $K$ & - & M10 \\
\hline 204211116 & $3947.6 \pm 2.7$ & $205.955 \pm 0.037$ & $7.240 \pm 0.032$ & $K_{\mathrm{p}}$ & 57584.38 & TW \\
\hline 204489514 & $3636.2 \pm 4.8$ & $86.759 \pm 0.074$ & $8.064 \pm 0.086$ & $K_{\mathrm{p}}$ & 57584.33 & TW \\
\hline 205238942 & $4176.0 \pm 2.2$ & $171.346 \pm 0.028$ & $4.332 \pm 0.004$ & $K_{\mathrm{p}}$ & 57225.29 & TW \\
\hline
\end{tabular}

${ }^{a}$ The starting epochs of the K2/C2 and K2/C15 light curves shown in Fig. 1 are MJD 56892 and 57988, respectively.

${ }^{b}$ B19, Barenfeld et al. (2019); M10, McClure et al. (2010); R05, Ratzka, Köhler \& Leinert (2005); TW, This work.

were organized into a series of sequential observing campaigns, which were limited to fields located around the ecliptic plane and to durations of roughly $80 \mathrm{~d}$. Quasi-periodic thruster firings throughout each observing campaign were then needed to correct for residual pointing drift, known as 'roll motion,' which caused characteristic 'sawtooth' patterns in the simple aperture photometry (SAP) light curves as targets moved around in their fixed apertures.

To correct for these effects, we use a modified pixel level decorrelation (PLD) method to remove the roll motion noise while preserving intrinsic, astrophysical variability. First, we use the INTERACT tool in the LIGHTKURVE package (Lightkurve Collaboration 2018) to hand-select pixel apertures, which are customized to include as much of the target flux as possible while avoiding nearby contaminants. We then sum the flux within these custom apertures, which are typically a few Kepler pixels across (where one Kepler pixel is 4 arcsec $\times 4$ arcsec) to build the SAP light curves. Decorrelation matrices are then generated from (1) pixel time series of neighbouring, quiet targets, which by nature strongly exhibit the $K 2$ roll motion pattern; (2) fourth-order polynomials in time to capture extremely long-term ( $>50 \mathrm{~d}$ ) variability due to changes in spacecraft temperature and velocity aberration; and (3) twodimensional, fourth-order polynomials of the point spread function (PSF) centroids in column and row (measured using LIGHTKURVE). We apply these decorrelation matrices in the same way as the PLD method from Luger et al. (2016), where optimum weights are derived for each component of the matrix using linear algebra. We split each light curve in half (at cadence number 97682) and fit the weights separately to each half; this accounts for a shift in the lightcurve noise properties, which is commonly seen in the $K 2$ data due to the change in Sun-angle on the spacecraft (and thus roll motion direction) at approximately the centre of each campaign. Along with the weights, we also simultaneously fit a Gaussian Process to the light curve in order to capture the astrophysical variability of the dippers, which is frequently orders of magnitude greater than the spacecraft systematics. The best-fitting decorrelation matrices are then weighted and summed to build our spacecraft motion correction. Using this method, any short-term variability due to spacecraft motion is removed, while astrophysical variability is preserved.

We note that for the two bright A-type stars in our sample (EPIC 204514548 and 204399980) the above method could not be applied due to saturation and flux bleed issues. Instead we use the publicly available $K 2$ Self Field Flattening (SFF) light curves described in Vanderburg \& Johnson (2014) and made available through the Mikulski Archive for Space Telescopes (https://archive.stsci.edu/kepler), which should be sufficient as the spacecraft motion noise is much smaller than the astrophysical trends for these bright stars. We also note that four dippers (EPIC 203770559, 203895983, 203936815, and 203950167) could not be separated from their bright, nearby companions (see Table 2) using aperture photometry given the large size of the Kepler pixels, although in some cases we detect both disc components around the primary and secondary; we discuss these candidate binary systems in Section 3.3.

Three dippers in our sample (EPIC 204281213, 204489514, and 204514548) also had a second epoch of $K 2$ data taken during Campaign $15(K 2 / \mathrm{C} 15)$, which was conducted $3 \mathrm{yr}$ after $K 2 / \mathrm{C} 2$. We plot the $K 2 / \mathrm{C} 15 \mathrm{SFF}$ light curves for these sources over their $K 2 / \mathrm{C} 2$ data in Fig. 1, illustrating how dipper behaviour can change on the time-scale of years. All three sources would still be classified as dippers based on the selection criteria applied in Section 2.1 when using their $\mathrm{K} 2 / \mathrm{C} 15$ light curves.

\subsection{Sub-mm/mm ALMA data}

The ALMA data used to resolve the dipper discs in our sample come from both our targeted ALMA programme as well as archival ALMA programmes, as described below. Data calibration and imaging are performed using CASA; the data were pipeline calibrated by NRAO staff and include standard flux, phase, bandpass, and gain calibrations. The ALMA Project IDs of the data used for each dipper are given in Table 1 .

Our targeted ALMA programme to resolve dipper discs (Project ID: 2016.1.00336.S; PI: Ansdell) was a high-resolution Band 6 $(\lambda \approx 1.3 \mathrm{~mm}$ ) survey of nine dippers conducted in Cycle 4 using the C40-7 configuration (21-3638 m baselines). The continuum spectral windows were centred on $233.29,220.40$, and $217.47 \mathrm{GHz}$ with bandwidths of 2.00, 2.00, and $1.88 \mathrm{GHz}$, respectively. The programme was split into two Science Goals, one for the four brighter $\left(F_{1.3 \mathrm{~mm}} \gtrsim 10 \mathrm{mJy}\right)$ dippers (EPIC 204630363, 203937317, 203843911, and 203824153) and another for the five fainter $\left(F_{1.3 \mathrm{~mm}}\right.$ $\approx 1 \mathrm{mJy}$ ) dippers (EPIC 204107757, 204489514, 204864076, 204757338, and 204211116) in the sample. The four brighter targets were observed on 2017 August 15 with $4512 \mathrm{~m}$ antennas and $4.7 \mathrm{~min}$ on-source integration times for a mean continuum rms of $0.07 \mathrm{mJy}_{\text {beam }}{ }^{-1}$. The five fainter targets were observed on 2017 Aug 18 with $4212 \mathrm{~m}$ antennas and $8.5 \mathrm{~min}$ on-source integration times for a mean continuum rms of $0.04 \mathrm{mJy}$ beam $^{-1}$. The 0.1 arcsec 
( $\sim 10 \mathrm{au})$ angular resolution of our observations was sufficient to resolve all the dipper discs targeted by our programme, except for those around EPIC 204107757 and 204757338, which were therefore not included in our sample.

We also make significant use of archival ALMA data, in particular those taken for the previously published large-scale surveys of Upper Sco (Carpenter, Ricci \& Isella 2014; Barenfeld et al. 2016, 2017) and $\rho$ Oph (Cieza et al. 2018; Williams et al. 2019). The $\rho$ Oph survey (Project ID: 2016.1.00545.S; PI: Cieza) was conducted in ALMA Band $6(\lambda \approx 1.3 \mathrm{~mm})$ during Cycle 4 and the sample was split into two Science Goals: the brighter and less evolved sources were observed at higher resolution $(0.25 \mathrm{arcsec})$ and sensitivity $\left(0.15 \mathrm{mJy}\right.$ beam $^{-1}$ continuum rms), while the fainter and more evolved sources were observed at lower resolution $(0.8$ arcsec $)$ and sensitivity $\left(0.25 \mathrm{mJy}^{\text {beam }^{-1}}\right.$ continuum $\left.\mathrm{rms}\right)$. The Upper Sco surveys were conducted in ALMA Band $7(\lambda \approx 880 \mu \mathrm{m})$ in Cycle 0 and Cycle 2 (Project IDs: 2011.0.00966.S, 2013.1.00395.S). The observations had angular resolutions between 0.35 and 0.73 arcsec with a median of 0.37 arcsec, and continuum rms values ranging from 0.13 to $0.26 \mathrm{mJy}_{\text {beam }}^{-1}$ with a median of $0.15 \mathrm{mJy}^{\text {beam }}{ }^{-1}$. Additionally, we make use of data taken for a new ALMA survey conducted to complete the (sub-)mm census of Upper Sco as new disc-hosting members have been discovered (Project ID: 2018.1.0056.S; PI: Carpenter); these data were taken in ALMA Band 7 during Cycle 6 with typical angular resolutions of 0.2 arcsec ( $\sim 25 \mathrm{au}$ ) and continuum rms values of $0.15 \mathrm{mJy} \mathrm{beam}^{-1}$.

We also use data from selected archival ALMA programmes when they offered higher spatial resolution. Thus, the data for EPIC 203850058, 204638512, and 204514548 come from ALMA programmes 2012.1.00046.S, 2017.1.01180.S, and 2013.1.00498.S with PIs Phan-Bao, Loomis, and Perez, respectively. Finally, although we do not perform model fits to these data in this work, four dippers in our sample (EPIC 203843911 or DoAr 25, EPIC 203860592 or WSB 52, EPIC 204281213 or HD 143006, and EPIC 204514548 or HD 142666) have higher resolution ( $\sim 5 \mathrm{au})$ ALMA observations taken as part of the Disc Substructures at High Angular Resolution Project (DSHARP; Andrews et al. 2018). In Section 3.2, we discuss these DSHARP data within the context of this work.

\subsection{Adaptive optics imaging}

All but two of the dippers in our sample (EPIC 204142243 and 205345560) have been inspected for close companions with highcontrast imaging (see Tables 2 and A1). Ten of these were observed and/or analysed as part of this work using adaptive optics (AO) imaging with the Near-infraRed imaging Camera (NIRC2) mounted on the $10 \mathrm{~m}$ Keck II telescope atop Maunakea. For the sources we observed with NIRC2, those with $R<13.5$ used natural guide star AO (Wizinowich et al. 2000; van Dam, Le Mignant \& Macintosh 2004), while the fainter sources used laser guide star AO (van Dam et al. 2006; Wizinowich et al. 2006). Imaging was done with the narrow camera and several sources also used non-redundant aperture masking (NRM).

For the NIRC2 data reduction, each frame is linearized and corrected for geometric distortion using the solution from Yelda et al. (2010), then the four image quadrants are used to de-bias the 'stripe noise' (i.e. spatially correlated readnoise) that results from electronics noise during readout being mirrored in each quadrant. Images are then dark-subtracted and flat-fielded using the most contemporaneous available calibration files, and 'dead' and 'hot' pixels are interpolated over. Dead pixels are identified from 'super- flats' taken in 2006-2013 as any pixel with a response $<30$ per cent in at least half of all super-flats. Similarly, hot pixels are identified from a comparable set of 'super-darks' as any pixel with $\geq 10$ counts in at least half of the super-darks. Pixels with flux levels $>10 \sigma$ above the median of the 8 adjacent pixels are flagged as cosmic rays or transient hot pixels and replaced with the median.

Our analysis of the standard AO imaging data broadly follows the methods of Kraus et al. (2016). To detect faint and wide ( $\gtrsim 500$ mas) companions, we subtract an azimuthal median PSF model. This adds no additional noise at wide separations, but leaves speckles in places, making it non-ideal for detecting close-in companions. To probe smaller inner working angles, we construct and subtract the best-fitting empirical PSF of another (single-star) target taken from among the 1000 images in the same filter nearest in time that were publicly available in the Keck Observatory Archive. In each individual frame, we measure the flux within 40 mas radius apertures centred on every image pixel, and compute the corresponding noise statistics as a function of radius by measuring the standard deviation of those fluxes in five-pixel-wide annuli around the primary. We then stack the detection-significance maps with a weighted mean, flagging any pixel with $>6 \sigma$ confidence as the location of a candidate companion.

Those candidates are then visually inspected to reject erroneous detections due to remaining cosmic rays and hot pixels as well as imperfect PSF subtraction of the strongest super-speckles. If any genuine companions are located within the speckle pattern of their primary star, the empirical PSF routine is repeated with a binary model that iteratively fits for the separation, position angle, and contrast of the two sources, then tests the reference PSFs (doubled using that binary model) to find the best-fitting empirical PSF template, repeated until the same best-fitting PSF template produces the same best-fitting binary model.

The analysis of the NRM observations broadly follows the methods of Kraus et al. (2008) and Ireland (2013). The NRM observations use a pupil plane mask to resample the telescope into a sparse interferometric array. This allows the use of the complex triple product, or closure-phase observable, to remove non-common path errors produced by atmospheric conditions or variable optical aberrations. To remove systematics in the closurephase observable, observations of the science targets are paired with calibration observations of other stars nearby in time, which were typically also science targets. Binary system profiles are then fit to the calibrated closure phases to measure component separations and position angles and calculate contrast limits.

The remaining dippers with high-contrast imaging were observed as part of previous surveys (see Tables 2 and A1). We defer to those works for details of the data reduction and analysis and adopt their reported results in this work.

\section{ANALYSIS}

\subsection{Outer disc inclinations}

Although many sources in our sample have disc inclinations derived from ALMA data reported in the literature (see Table 1), these were derived with disparate methods, and there are some inconsistencies among reported values. For example, the disc inclinations for $\rho$ Oph targets from Cieza et al. (2018) and Cox et al. (2017) must be inferred from the reported semimajor and semiminor axes of 2D Gaussian model fits to the observed visibilities using CASA's uvmodelfit task. The disc inclinations of Upper Sco members from Barenfeld et al. (2017), on the other hand, use a 
Markov chain Monte Carlo (MCMC) approach to fit the observed visibilities to synthetic visibilities derived from a self-consistent disc model with an assumed dust surface density profile parametrized by a truncated power law. The latter method is more physically motivated, but can be limiting for moderate signal-to-noise and/or marginally resolved discs, which are common in large-scale ALMA disc surveys. However, the former method is not appropriate for discs with large inner cavities (there are a few of these 'transition discs' in our sample; see Fig. 1) and also does not provide posterior distributions that are useful for our analysis of the disc inclination distribution (see Section 4.1).

Thus, we (re)derive the disc inclinations for our entire sample using the GALARIO computational library (Tazzari, Beaujean \& Testi 2018) combined with the EMCEE package (Foreman-Mackey et al. 2013), which allows us to quickly fit 2D disc models to the ALMA visibilities by enabling the rapid exploration of parameter space. GALARIO is a PYTHON library that uses GPUs, or alternatively multiple CPU cores, to speed up the computation of synthetic visibilities. Because of its modular structure, we can use GALARIO for the likelihood computation in EMCEE, a PYTHON implementation of MCMC Ensemble sampling for Bayesian parameter estimation. We use the GPU version of GALARIO, which is $\sim 150 \times$ faster than standard PYTHON implementations that rely on SCIPY and NUMPY packages. Tazzari et al. (2018) provides a detailed explanation of employing GALARIO to fit interferometric visibilities like those from ALMA and we follow their general implementation procedure. The fits typically use 120 walkers and 5000 steps for the MCMC; the mean accepted fraction of steps are all between 0.2 and 0.5 , implying that the chains are converging.

Most of the dippers in our sample are single stars whose discs lack resolved structure (e.g. no gaps or rings), thus we most often fit the visibilities using a simple 2D Gaussian disc model with six free parameters: a flux normalization term, the full width-half-max along the semimajor axis, the inclination $\left(i_{\mathrm{d}}\right)$ and position angle $\left(\mathrm{PA}_{\mathrm{d}}\right)$ of the disc, and the offset in right ascension and declination of the source centre from the phase centre. For the two resolved binary discs in our sample (EPIC 203770559 and 203936815), we simultaneously fit two 2D Gaussians to the data. Four dippers (EPIC 203950167, 204630363, 204638512, and 205238942) exhibit large inner cavities; for these, we use the aforementioned 2D Gaussian model, but with one more free parameter, an inner cut-off radius. EPIC 203843911 and EPIC 204281213 exhibit more complex features in the data that our simple models could not account for (i.e. narrow gaps/rings and azimuthal asymmetries, respectively; see Fig. 1). However, in both cases our inclinations agree well with those derived by Huang et al. (2018) from the high-resolution DSHARP observations of these objects.

Indeed, for all four dippers in our sample that overlap with DSHARP (see Fig. 2), our derived disc inclinations match to within $\sim 1^{\circ}$ of those from Huang et al. (2018). This instills general confidence in the precision of our inclinations, which are derived from simple models of more moderate resolution data. This is consistent with the general findings of Huang et al. (2018), who performed both simple 2D elliptical Gaussian fits to the DSHARP discs as well as more detailed fits to the individual well-resolved annular substructures within each disc, finding that the derived inclinations agreed to within roughly a degree in all cases, with no apparent biases in the results. This suggests that any effects from fitting the simple 2D Gaussian models in this work (e.g. due to unaccounted model-dependent uncertainties) is on the level of $\lesssim 1^{\circ}$ and thus should not impact our analysis, which focuses on population-level statistics.

\subsection{Disc morphologies}

Fig. 1 shows that dipper discs exhibit a wide range of morphologies, from compact discs (e.g. EPIC 203937317) to extended discs (e.g. EPIC 203843911) to discs with large inner cavities (e.g. EPIC 204638512), and even discs with azimuthal asymmetries (e.g. EPIC 204281213). Although many dipper discs appear featureless at the current spatial resolution, the growing number of discs with very high spatial resolution ALMA data (i.e. at scales of a few au; ALMA Partnership et al. 2015; Andrews et al. 2016, 2018) suggest that most discs will exhibit structure (most commonly concentric rings and gaps; Huang et al. 2018) if observed at sufficiently high spatial resolution.

Four of the dippers in our sample overlap with DSHARP, a programme that mapped the millimetre continuum of 20 protoplanetary discs at spatial resolutions of $\sim 5$ au (Andrews et al. 2018). The DSHARP images of these four dippers are shown in Fig. 2. Huang et al. (2018) performed a systematic analysis of the annular substructures (i.e. the bright and dark annuli) visible in the DSHARP discs, finding a range of morphologies and no clear trends in disc architecture within their sample. With regards to the four dippers in our sample, they found that EPIC 204281213 (HD 143006) hosts a disc with complex structure, exhibiting three bright rings (centred at 6,41 , and $65 \mathrm{au}$ ) and two gaps (centred at 22 and $51 \mathrm{au}$ ) as well as a bright crescent at $80^{\circ}<\theta<144^{\circ}$ and potential inner cavity. In stark contrast, EPIC 203860592 (WSB 52) hosts a relatively compact disc with only one low-contrast ring and gap (centred at 25 and $21 \mathrm{au}$, respectively) as well as an optically thick core that extends out to nearly $30 \mathrm{au}$. The disc around EPIC 204514548 (HD 142666) shows four bright rings (centred at 6, 20, 40, and $58 \mathrm{au}$ ) and three gaps (centred at 16,37, and $55 \mathrm{au}$ ) as well as a potential inner cavity surrounded by an inner disc brightness asymmetry, which may be due to viewing the heated and puffed-up interior of a ring. Finally, EPIC 203843911 (DoAr 25) hosts a particularly extended disc with three faint rings (centred at 86,111, and $137 \mathrm{au}$ ) and three gaps (centred at 74, 98, and $125 \mathrm{au}$ ) around a bright (but not very optically thick) core.

The morphological diversity of these four dipper discs appears to echo that seen in the overall DSHARP sample, with no obvious shared traits. Just as many exhibit potential inner cavities as those that do not (although the sample size is small), and while all have some sort of ringed structure, this seems to be a common feature among the general disc population when observed at sufficiently high spatial resolution. Moreover, the morphology of the ringed structure is diverse among the dipper sample, reflecting what is also found in the larger DSHARP sample. We note that four other DSHARP discs (Elias 20, Elias 24, Elias 27, and AS 205) have $K 2 / \mathrm{C} 2$ light curves but do not exhibit dipper behaviour.

\subsection{Stellar multiplicity}

Many stars are in multiple systems (see review in Duchêne \& Kraus 2013) and blending in the $K 2 / \mathrm{C} 2$ light curves is a concern due to the large Kepler pixel sizes ( 4 arcsec $\times 4$ arcsec or 520 au $\times 520$ au at the distance of our sample). This risks complicating the interpretation of our inclination results, if more than one component could be the source of the dipper signal in the $K 2$ data or could host a disc that remains undetected and/or unresolved in our ALMA observations. Fortunately, all but two of the dippers in our sample have been surveyed for close companions with high-contrast imaging (see Section 2.4). Eight were found to have candidate close ( $\lesssim 5$ arcsec) companions; Table 2 gives the separations $(\rho)$, position angles (PA), and contrasts $(\Delta m)$ of the detected candidate companions. For all 

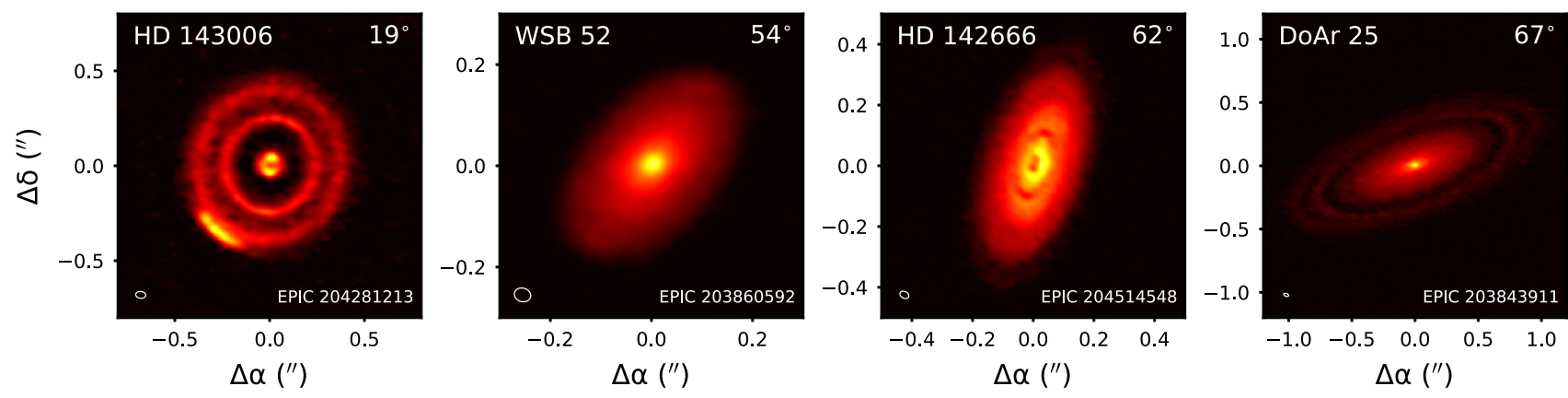

Figure 2. High-resolution ( $\sim 5 \mathrm{au})$ ALMA images of the four dippers in our sample included in DSHARP. Names and inclinations from DSHARP (Huang et al. 2018) are given at the top of each panel, EPIC numbers are shown in the lower right, and the beam size is illustrated by the white ellipse in the lower left.

sources in our sample with high-contrast imaging, detection limits as a function of separation from the primary, when available, are given in Table A1. Moreover, we can use information from the Gaia Data Release 2 (DR2) as an independent check of binarity and also to assess the likeliness of any candidate companions being physically bound. The latter could be of interest when interpreting the dipper phenomenon and has implications as to whether the potential contaminants remain blended in the $K 2$ light curves taken at different epochs.

The imaged candidate companions to four of the dippers in our sample (EPIC 203843911, 204211116, 204489514, 205238942) are sufficiently separated and/or faint that they may be older background objects (e.g. see discussion in Barenfeld et al. 2019) and indeed we do not detect discs at the locations of their candidate companions in our ALMA data. The Gaia DR2 proper motions and parallaxes of EPIC 204489514 and its brighter candidate companion do not match and thus these sources are likely unassociated (the fainter candidate companion is not detected in Gaia DR2). Although EPIC 205238942 and its candidate companion have similar parallaxes and proper motions, making them possible true companions, the secondary's faintness rules it out as the source of the dipping (but also makes it an interesting potential low-mass brown dwarf companion of $\sim 30-50 \mathrm{M}_{\text {Jup }}$ at $\sim 600 \mathrm{au}$ ). The candidate companions to EPIC 204211116 and 203843911 are not detected in Gaia DR2, likely due to their faintness, and their large separations make them unlikely to be associated with the dippers.

Three of the remaining imaged candidate companions (to EPIC 203770559, 203936815, 203950167) are sufficiently close that they may be gravitationally bound, but also sufficiently separated that their discs may have avoided substantial tidal truncation (e.g. Harris et al. 2012), and indeed we detect discs around both components in two of these cases (EPIC 203770559 and 203936815). We do not detect a disc around the secondary to EPIC 203950167, and Gaia DR2 gives marginally inconsistent parallaxes (3-4 $\sigma$ differences) and similar but inconsistent proper motions (1-3 mas $\mathrm{yr}^{-1}$ differences); regardless, the faint companion, although blended in the $K 2 / \mathrm{C} 2$ light curve, is unlikely to be the source of the dipping, as the photometric variability is larger than the primary-secondary flux ratio.

The remaining dipper with an imaged candidate companion (EPIC 203895983) is in a close, nearly equal-mass system. Although we do not clearly resolve two discs in our ALMA data, the imaged disc is noticeably asymmetric compared to our overall sample (see Fig. 1) and the elongation is along the same PA as the binary. This suggests that there are two discs that are blended in our ALMA data and that the derived inclination should not be trusted.
An independent check for binarity comes from the Gaia DR2 astrometric fits (Lindegren et al. 2018) through the re-normalized unit weight error (RUWE). RUWE measures the goodness-of-fit (similar to reduced $\chi^{2}$ ) of the Gaia DR2 astrometric solution compared to stars of similar colour and brightness. Large RUWE values $(>1.4)$ are indicative of an unresolved companion impacting the solution to the DR2 astrometry (e.g. Ziegler et al. 2019). The two dippers in our sample lacking AO data (EPIC 204142243 and 205345560) have only moderate RUWE values (0.93 and 1.26, respectively) consistent with being single stars. EPIC 204638512 is the only dipper in our sample with RUWE $>1.4$, suggesting a companion too close and/or too faint to be detected by the current AO data (see Table A1). Interestingly, this dipper hosts a disc with a large inner cavity whose properties are consistent with being carved by a massive planet (e.g. Pinilla et al. 2018b). Although nebulosity around young stars could also increase their RUWE values (e.g. Long et al. 2019), EPIC 204638512 is located in the evolved Upper Sco region and has no evidence for surrounding nebulosity. Finally, EPIC 203895983 is the one star in our sample without a Gaia DR2 parallax, which is also a potential indicator of binarity, as parallaxes are only reported if a single-star solution is found; indeed, as discussed above, this source is a near-equal-mass binary in the AO data with a likely blended binary disc in the ALMA data.

Thus, the dippers in our sample that may be of concern from a multiplicity standpoint are EPIC 203770559 and 203936815, for which we detect both discs in our ALMA observations, and EPIC 203895983, whose disc components are likely blended in our ALMA data. We flag these systems in the remaining analysis throughout this paper.

\section{DISCUSSION}

\subsection{Outer disc inclination distribution}

Fig. 3 shows the distribution of dipper disc inclinations resolved by ALMA (Section 3.1; Table 1). For the two resolved binary discs (EPIC 203770559 and 203936815), we use the inclination of the disc around the primary, but also show the inclination distribution when removing these sources as well as the likely blended binary disc (EPIC 203895983) from the sample (see Section 3.3). In either case, the distribution appears approximately uniform with $\cos i_{\mathrm{d}}$ out to $i_{\mathrm{d}} \approx 75^{\circ}$, suggesting an isotropic disc inclination distribution, with the exception of the most highly inclined cases.

The deficit of highly inclined systems is an expected observational bias, as these edge-on discs will obscure their host stars with 


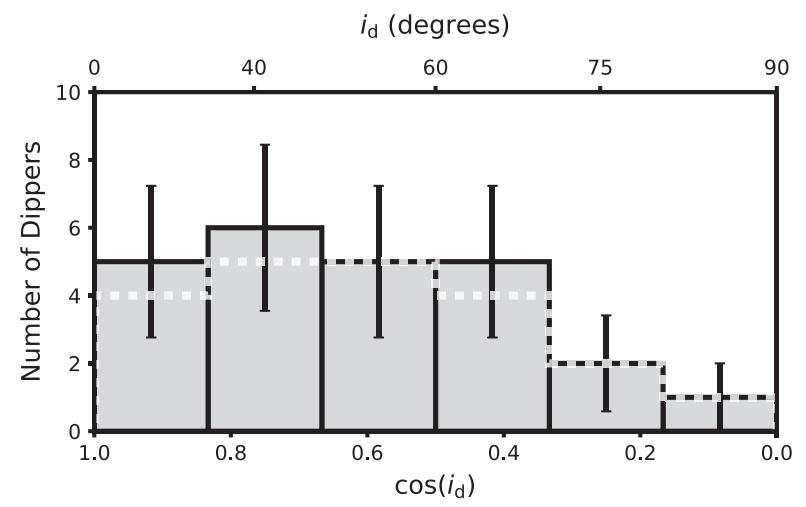

Figure 3. The inclination distribution of dipper discs resolved by ALMA, where an isotropic distribution is flat in $\cos \left(i_{\mathrm{d}}\right)$. The disc inclination values are taken from Table 1. For the two resolved binary disc systems, we use the inclination of the disc around the primary. The error bars are the square root of the number of dippers in the histogram bins. The white dashed line shows how the results would change if the two resolved binary discs and likely blended binary disc are removed (see Section 3.3).

their optically thick mid-planes, making the stars too faint to be included in the $K 2$ target catalogue. To confirm this quantitatively, we generated a grid of protoplanetary disc models using MCFOST (Pinte et al. 2006), a 3D Monte Carlo radiative transfer code that simulates images of discs at a given wavelength for specified disc structure and dust grain properties (see Appendix B for details of the model grid). Using our MCFOST model grid, we confirm that $\gtrsim 50$ per cent of stars with discs of $i_{\mathrm{d}} \gtrsim 80^{\circ}$ would be undetected by $K 2$, rising to $\gtrsim 90$ per cent at $i_{\mathrm{d}} \gtrsim 85^{\circ}$ (Angelo et al., in prep), consistent with Fig. 3. This supports the interpretation that the low dipper occurrence rate at high disc inclination is an observational bias.

In order to more robustly test whether the inclination distribution at $i_{\mathrm{d}} \lesssim 75^{\circ}$ is consistent with isotropic, we construct an empirical cumulative distribution function (ECDF), which is a nonparametric estimator of the cumulative distribution function for a random variable. We build the ECDF by randomly sampling from the inclination posterior distributions inferred with GALARIO for each dipper, converting these to $\cos \left(i_{\mathrm{d}}\right)$, then applying the ECDF package in the STATSMODEL PYTHON module. We then repeat this 1000 times and take the mean and standard deviation as the final values and associated uncertainties of the ECDF. The result is shown in Fig. 4 and compared to the isotropic case, constructed by randomly sampling from a uniform distribution between 0.26 and 1.0 for $\cos \left(i_{\mathrm{d}}\right)$ (corresponding to $0<i_{\mathrm{d}}<75^{\circ}$ due to the observational bias discussed above) for each dipper in our sample (i.e. the sample sizes are the same). The distributions clearly overlap, demonstrating that the dipper disc inclination distribution is consistent with isotropic. The average and standard deviation of the $p$-values of two-sided Kolmogorov-Smirnov tests, calculated for each of the 1000 draws and only considering $\cos \left(i_{\mathrm{d}}\right)>0.26$, is $0.64 \pm 0.27$, indicating that we cannot reject the null hypothesis that the dipper $\cos \left(i_{\mathrm{d}}\right)$ distribution and the uniform distribution are drawn from the same parent distribution (this result holds even when removing the three binary discs).

Our findings are in contrast to those of Cody \& Hillenbrand (2018), who reported that dipper discs favour higher inclinations $\left(i_{\mathrm{d}}\right.$ $\gtrsim 50^{\circ}$ ) apart from a few face-on exceptions. They used a similarly selected sample (i.e. dippers identified by their $K 2 / \mathrm{C} 2$ light curves with disc inclinations derived from resolved ALMA data), however

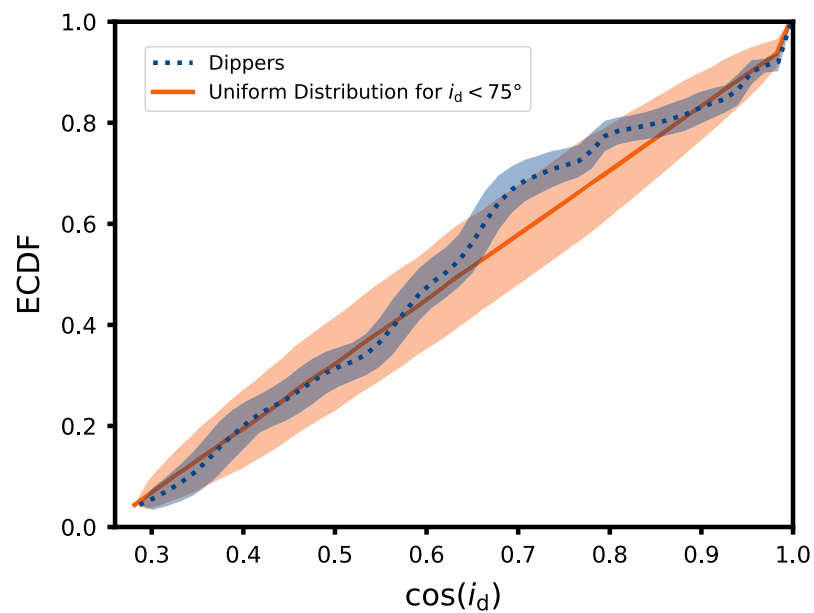

Figure 4. The ECDF of the dipper disc inclinations resolved by ALMA (blue dashed line) compared to a uniform distribution for $i_{\mathrm{d}}<75^{\circ}$ (solid orange line); the shaded regions represent $1 \sigma$ uncertainties (see Section 4.1). We only consider $i_{\mathrm{d}}<75^{\circ}$ for the uniform distribution as more edge-on discs will block their star, creating an observational bias seen in the observed dipper sample (see Section 4.1 and Fig. 3). These two distributions being statistically indistinguishable suggest that dippers have an isotropic outer disc inclination distribution.

their disc inclinations were taken directly from the literature rather than being uniformly analysed and no formal statistical tests were reported. Moreover, many of the highly inclined discs used in Cody \& Hillenbrand (2018) were taken from Barenfeld et al. (2017) and have particularly large uncertainties $\left( \pm 40-60^{\circ}\right)$. In Appendix C, we present these dipper discs with large uncertainties and show that including them in our samples only makes the dipper disc inclination distribution even more consistent with isotropic by filling in the high inclination end of the distribution.

Finally, we note that four sources in our sample are A-type or G-type stars (EPIC 204281213, 203950167, 204399980 and 204514548; see Table 1). Although earlier-type stars exhibiting dimming events are often classified as UXOR variables, the photometric variability of dippers and UXORs are distinctly different: UXORs are characterized by deep (up to several magnitudes), long-term (weeks to years) dimming events while dippers are characterized by shorter (days) and relatively shallower (a few tens of percent) dimming events. Moreover, there are now several examples of young stars exhibiting both dipper and UXOR phenomena over time (namely the prototypical dipper AA Tau, which is currently undergoing a UXOR-type dimming event; Bouvier et al. 2013). Nevertheless, removing these four sources from our sample does not change our overall results, as their disc inclinations are evenly distributed.

\subsection{Correlations with light curve and disc properties}

Different mechanisms to explain the dippers (e.g. accretion-related inner disc warps versus disc winds; see Section 4.3) may produce light curves with different morphologies and be more likely to occur at different disc inclinations. Fig. 5 therefore graphically represents the dipper disc inclinations, plotted in the light curve morphology space, as represented by the flux asymmetry $(M)$ and quasiperiodicity $(Q)$ statistics defined in Cody et al. (2014). According to these statistics, light curves with symmetric flux distributions about a median amplitude (e.g. sinusoidal star-spot patterns) have 


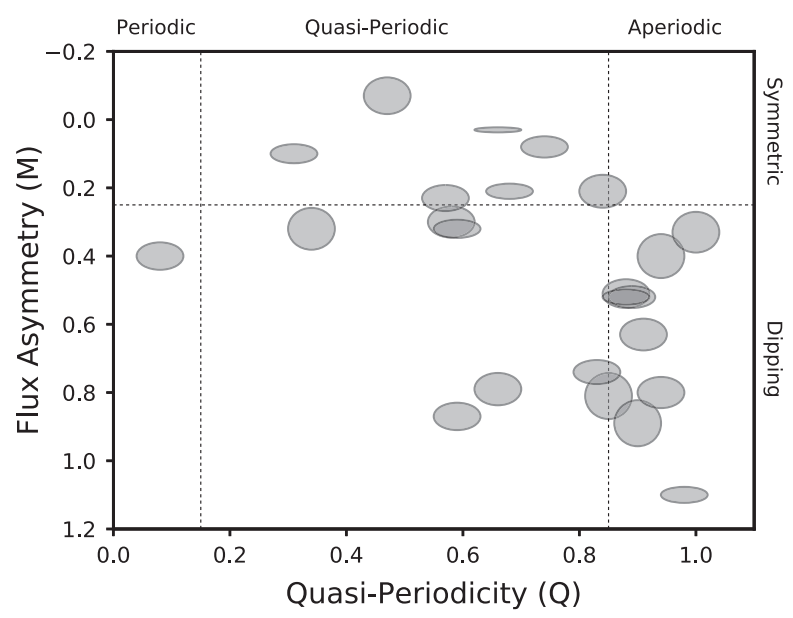

Figure 5. Graphical representation of dipper disc inclinations, distributed in a plot of flux asymmetry $(M)$ versus quasi-periodicity $(Q)$, as defined in Cody et al. (2014) and calculated in Cody \& Hillenbrand (2018). The dashed lines are the delimiters of the different types of light curve morphologies, identified by eye in Cody \& Hillenbrand (2018). There is no perceptible pattern in dipper disc inclination with variability type (see Section 4.1).

$M=0$, and more negative-going light curves (e.g. dippers) have higher positive $M$ values; perfectly periodic light curves have $Q=$ 0 , while those with $Q \approx 1$ are aperiodic. Fig. 5 illustrates that there is no perceptible correlation between disc inclination and light curve morphology, at least as defined by the $Q$ and $M$ statistics of Cody et al. (2014). The lack of low $Q$ and high $M$ sources (i.e. quasi-periodic dippers with large negative deviations relative to the median) should not be interpreted as the quasi-periodic dippers tending to have shallow dips; rather, this is likely due to quasiperiodic dippers often being dominated by star-spot patterns with high duty cycles (e.g. EPIC 203860592, furthest left in Fig. 5), which drives the median of the light curve to more negative values, and thus the $M$ statistic to smaller positive values.

One explanation for this lack of correlation is that the photometric behaviour of dippers is known to change over year-long (and possibly shorter) time-scales. McGinnis et al. (2015) found that some dippers in NGC 2264, observed by CoRoT in 2008 and then again in 2011, switched between aperiodic and quasi-periodic variability (or vice versa) at some point between the two epochs (and possibly more than once). Significant changes in dipper light curve morphology on similar time-scales are also seen when comparing the $K 2 / \mathrm{C} 2$ and $K 2 / \mathrm{C} 15$ light curves in Fig. 1 for the three sources in our sample that were observed in both campaigns, which were separated by $3 \mathrm{yr}$. In contrast, the prototypical dipper AA Tau maintained a clear quasi-periodic dipping pattern for at least $20 \mathrm{yr}$ (Bouvier et al. 2013). Building up a larger sample of dippers with multiple epochs of high-precision photometry are needed to investigate this possibility, and this should become possible in the near future, for example by combining the $K 2$ and Transiting Exoplanet Survey Satellite (TESS; Ricker et al. 2014) data sets.

To investigate correlations between disc properties and disc inclination, we plot mid-infrared excesses (relative to the expected stellar photosphere values) in the Wide-field Infrared Survey Explorer (WISE; Wright et al. 2010) W2 $(4.6 \mu \mathrm{m}), W 3(12 \mu \mathrm{m})$, and $W 4(25 \mu \mathrm{m})$ bands against $i_{\mathrm{d}}$ in Fig. 6. These excesses are calculated as in Luhman \& Mamajek (2012), and we also follow
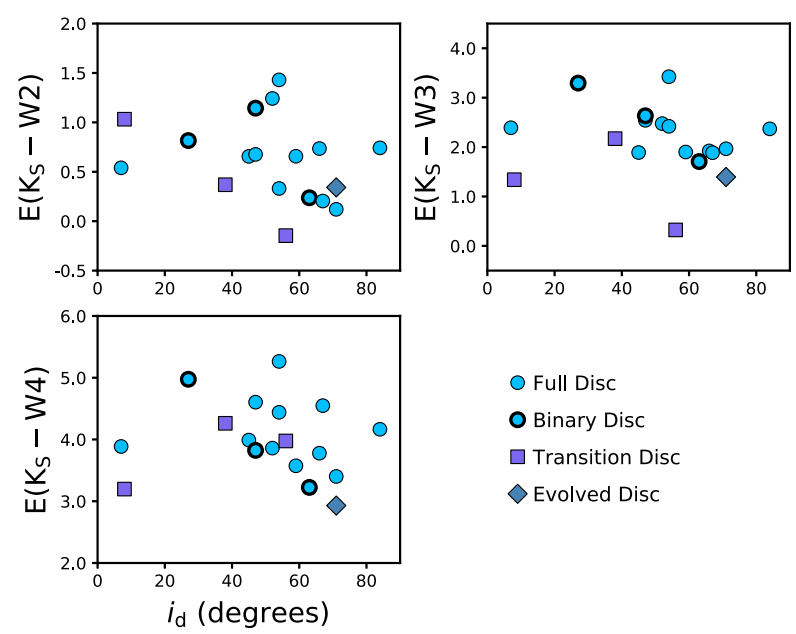

O Full Disc

O Binary Disc

$\square$ Transition Disc

$\diamond$ Evolved Disc

Figure 6. WISE mid-infrared excess over the stellar photosphere versus disc inclination for the late-type (K/M) dippers in our sample (see Section 4.2). Circles are 'full' discs, squares are 'transition' discs with large inner dust cavities, and diamonds are 'evolved' discs. Those with thicker outlines are the three binary discs discussed in Section 3.3.

their procedure for using these excesses to classify disc type (e.g. full, transitional, evolved; see fig. 6 in Ansdell et al. 2016b). We note that these methods use spectral type as a reference for the stellar photosphere, and that the spectral types used in this work are taken from inhomogeneous literature sources (see Table 1), thus this analysis should be repeated once homogenous spectral types are derived for our sample. The $W 2$ wavelength corresponds to peak blackbody emission at $\sim 600 \mathrm{~K}$, which is the expected temperature of dust grains orbiting at $\sim 10$ stellar radii around these pre-main sequence stars, while the $W 3$ and $W 4$ emission probe cooler dust within an au to a few au. Thus, the amount of WISE excess might be expected to depend on disc viewing geometry: all else being equal, a highly inclined, optically thick disc will subtend a smaller solid angle and produce a smaller mid-infrared excess. Moreover, at very high inclinations, the inner regions of a flared disc will become (partially) obscured, attenuating emission at near-infrared wavelengths.

We find no statistically significant correlations between disc inclination and WISE excess (Spearman rank tests return $\rho=$ $-0.37,-0.31$, and -0.25 with $p$-values of $0.13,0.21$, and 0.32 for the $W 2, W 3$, and $W 4$ excesses, respectively). None the less, Fig. 6 does hint towards decreasing W4 excess with higher disc inclinations, as expected from the above geometric arguments. This suggests that disc material at separations $(\sim 2 \mathrm{au})$ and temperatures $(\sim 100 \mathrm{~K})$ that correspond to peak emission at $25 \mu \mathrm{m}$ is co-inclined with the outer ( $\gtrsim 10 \mathrm{au}$ ) disc resolved by ALMA. The two exceptions have the lowest inclinations: EPIC 20397137 (DoAr 24), a very compact disc, and EPIC 204638512 (J1604), a transition disc with a thin annulus, both of which may lack material at $\sim 2$ au. The absence of a correlation between $W 2$ excess and disc inclination may be explained by the structure of the inner $(\lesssim 1 \mathrm{au})$ disc being completely unrelated to the geometry of the outer disc resolved by ALMA. Moreover, emission at these shorter wavelengths can be variable: a striking example is EPIC 204638512 (J1604), whose Spitzer/IRAC photometry (taken in the early 2000s) shows no infrared excess at $\lesssim 10 \mu \mathrm{m}$, while its WISE photometry (from 2010) and Spitzer/IRS spectra (from 2007) show 

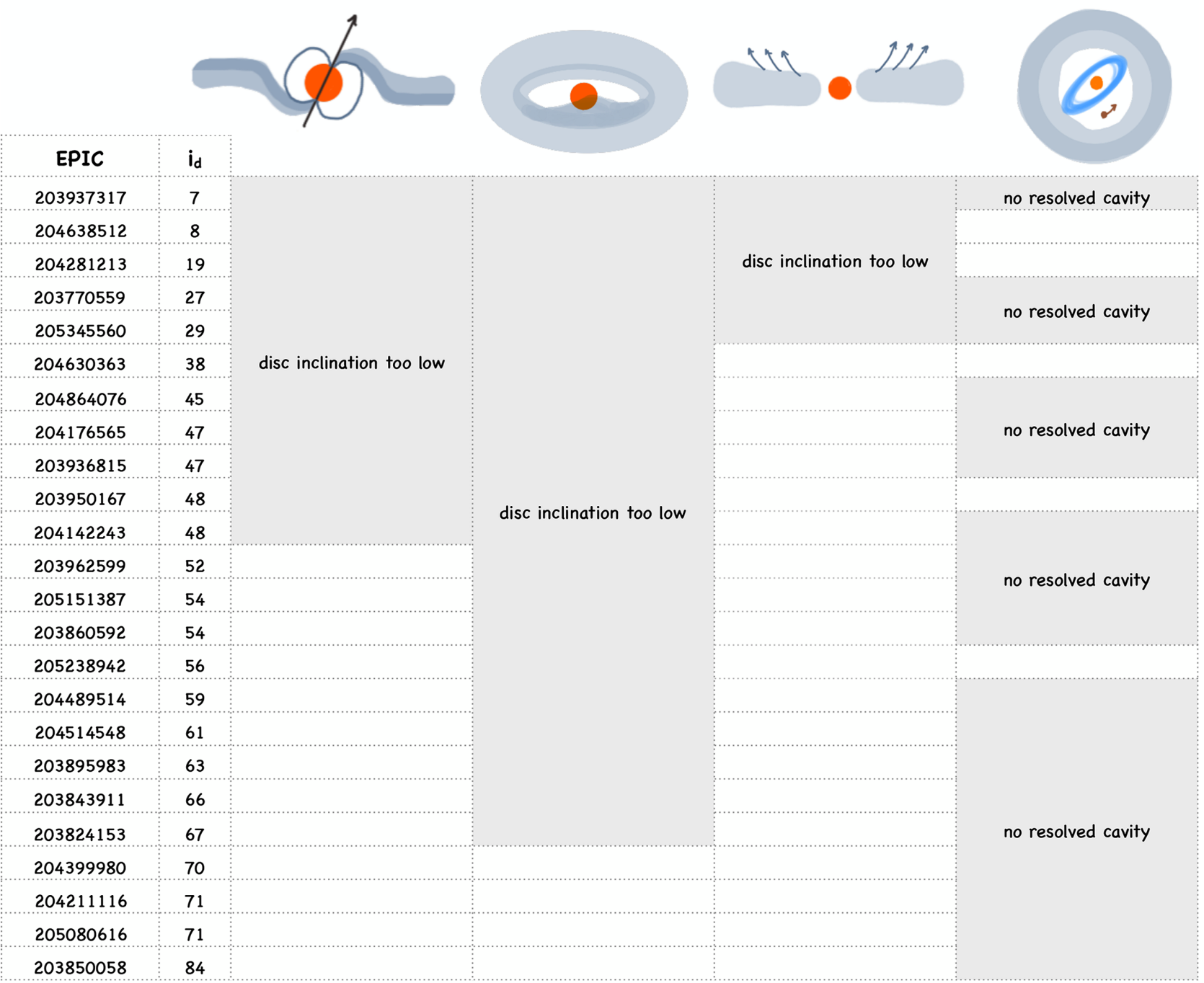

Figure 7. Proposed dipper mechanisms and their feasibility for the dippers in our sample given our derived outer disc inclinations. Note that for the 'accretion driven inner disc warp' scenario, we consider inclined stellar magnetic fields that allow for more moderate disc inclinations (Bodman et al. 2017). Moreover, for the 'broken inner disc' scenario, future ALMA observations at higher spatial resolution may reveal smaller inner cavities not yet resolved.

significant excess at these same wavelengths (e.g. see fig. 4 in Zhang et al. 2014).

\subsection{Expectations from theory}

Multiple mechanisms have been considered to explain the dippers, and they often require or are biased towards specific viewing geometries. Therefore, in this section, we compare the expectations from these theories to our observed disc inclination distribution, as summarized in Fig. 7.

One of the first mechanisms that was crafted to explain the dipper phenomenon - and in particular the $8.5 \mathrm{~d}$ quasi-periodic photometric variability of the prototypical dipper AA Tau - is highly non-axisymmetric, magnetically funnelled accretion from the inner disc edge on to the star, creating an inner disc warp that partially occults the star as the disc rotates (Bouvier et al. 1999). This 'accretion-driven inner disc warp' scenario requires nearly edge-on disc inclinations ( $\sim 70^{\circ}$; e.g. see McGinnis et al. 2015; Kesseli et al. 2016), a viewing geometry supported for AA Tau by studies of polarization (Ménard et al. 2003) and emission line shape (Bouvier et al. 2003), though disputed by ALMA observations that clearly show a moderately inclined outer disc $\left(59.1 \pm 0.1^{\circ}\right.$; Loomis et al. 2017). This mechanism could still explain the dipping behaviour of AA Tau, however, as Bodman et al. (2017) used magnetospheric truncation theory to show that, if the stellar magnetic field axis is sufficiently tilted, this mechanism could be extended to moderate disc inclinations. However, unlike AA Tau, most dippers have accretion rates that are below the classical $\mathrm{T}$ Tauri level (Ansdell et al. 2016b), making the accretion-driven disc warp scenario unlikely in most cases, especially for the aperiodic dippers that require high accretion rates to drive unstable accretion regimes (Kurosawa \& Romanova 2013). Moreover, it is telling that of the four most highly inclined $\left(i_{\mathrm{d}} \gtrsim 70^{\circ}\right)$ dippers in our sample, only EPIC 204211116 has an AA Tau-like (i.e. quasi-periodic) light curve, while there are multiple examples at moderate inclinations (see Fig. 1).

Another explanation for the dippers that requires nearly edge-on viewing geometries is occultations of the star by vertical structures 
produced by some instability in the disc. One incarnation of this 'vertical disc instability' scenario involves vortices produced by Rossby waves, which occur at an extremum in the disc vortensity (i.e. the ratio of the vorticity to density; Lovelace et al. 1999; Meheut, Yu \& Lai 2012a; Meheut et al. 2012b, 2013; Lin 2013; Ono et al. 2016), such as at the boundary of a 'dead zone' where the magnetorotational instability ceases to operate (Lyra \& Mac Low 2012; Miranda, Lai \& Méheut 2016; Miranda et al. 2017). These structures will be limited to 1-2 scale heights above the disc; at the characteristic temperatures $(\sim 1000 \mathrm{~K})$ and orbital periods $(\sim 4 \mathrm{~d})$ where the structures would have to be located to explain the dippers (i.e. assuming $\sim 10 \mathrm{Myr}$ old K/M-type stars with the occulting dust orbiting near the star-disc co-rotation radius; Ansdell et al. 2016b; Bodman et al. 2017), the scale height would be $\sim 0.1$ au and thus the disc inclinations would be limited to $i_{\mathrm{d}} \gtrsim 70-80^{\circ}$. This rules out the vertical disc instability scenario for most of the dippers, if the inner disc where these instabilities occur has the same geometry as the outer disc resolved by ALMA.

One mechanism for the dippers could involve dust being lofted in disc winds driven by stellar XUV radiation and/or impelled by radial magnetic fields (Blandford \& Payne 1982). Dust clouds crossing our line of sight can contribute to both photometric variability and a 'bump' in infrared emission near $3 \mu \mathrm{m}$ (Tambovtseva \& Grinin 2008; Bans \& Königl 2012). This 'dusty disc wind' scenario could also explain emission from silicates well above the disc mid-plane (Varga et al. 2017; Giacalone et al. 2019), the infrared variability of MWC 480 (Fernandes et al. 2018), and contemporaneous optical dimming and infrared brightening of HD 163296 (Ellerbroek et al. 2014). However, magnetized disc winds are limited to angles $\gtrsim 30^{\circ}$ from pole-on (Blandford \& Payne 1982), and if we assume the occultation occurs near the wind launch radius, then we should expect a deficit of nearly face-on dipper discs. This is also not seen in our observed distribution, although obscuration by associated jets could be responsible for systems with the lowest inclinations.

One possibility, of course, is that there are significant misalignments between the outer discs resolved by ALMA and the unseen inner discs. Directly constraining the geometry of inner discs is difficult as it requires near-infrared interferometry and thus is only possible for bright (and thus typically high-mass) sources. There are a couple of bright UXOR objects with resolved outer disc geometries whose inner $(<1 \mathrm{au})$ disc inclinations have also been constrained by near-infrared interferometry: the archetype UX Ori has an inner and outer disc inclination of $\sim 70^{\circ}$ revealed by near- and mid-infrared interferometry, respectively (Kreplin et al. 2016); CQ Tau has an inner disc inclination of $\sim 48^{\circ}$ constrained by near-infrared interferometry (Eisner et al. 2004) while ALMA measures an outer disc inclination of $\sim 37^{\circ}$ (Pinilla et al. 2018a). For these sources, the inner and outer discs appear fairly aligned. However, this is expected for UXORs, as their variability has been successfully explained by puffed-up inner disc rims that also selfshadow their outer discs, causing their observed weak far-infrared excesses (Dullemond et al. 2003). Unfortunately, most inner discs cannot be resolved for the typically late-type (and thus faint) dipper stars, although one of the higher mass dippers in our sample, EPIC 204514548 (HD 142666), has an outer disc inclination of $61^{\circ}$ measured by ALMA and an inner disc inclination of $\sim 58^{\circ}$ constrained with CHARA (Davies et al. 2018).

Misalignments can be indirectly detected, however, via the shadows that an inclined inner disc casts on the outer dust disc in high-contrast optical/infrared images (e.g. Marino et al. 2015; Min et al. 2017; Pinilla et al. 2018b) or by velocity perturbations imprinted in the gas kinematics (e.g. Teague et al. 2018). Velocity perturbations in $\mathrm{HCO}^{+}$emission have been seen for the dipper AA Tau (Loomis et al. 2017), and both kinematic signatures in the gas (Mayama et al. 2018) and variable shadows in scattered light (Takami et al. 2014; Pinilla et al. 2018b) have been observed for EPIC 204638512 (J1604). For the latter, Davies (2019) also derive a stellar inclination that is highly misaligned with the outer disc.

These misaligned inner discs could be induced by stellar companions (e.g. Facchini, Juhász \& Lodato 2018) or planets with masses down to $1 M_{\text {Jup }}$ (e.g. Matsakos \& Königl 2017; Nealon et al. 2019; Zhu 2019) on orbits inclined relative to the outer disc. Simulations show that when these companions open a gap, the disc inside the orbit breaks from the outer regions and becomes misaligned. The inner discs can be highly misaligned with respect to the outer disc (i.e. $\gtrsim 70^{\circ}$; Min et al. 2017; Facchini et al. 2018), producing pairs of narrow shadows in the outer disc that can be highly dynamic. Indeed, Pinilla et al. (2018b) observed with VLT/SPHERE (Beuzit et al. 2019) that a pair of narrow shadows on the outer disc around one of the face-on dippers in our sample, EPIC 204638512 (J1604), were variable both in morphology and in position on time-scales of days. Even moderately inclined planet orbits $\left(\sim 10^{\circ}\right)$ can misalign inner discs and cast shadows on the outer regions (e.g. Nealon et al. 2019), although these shadows should be broad in extent (rather than narrow lanes), and could explain discs observed to be covered up to half in shadow (e.g. Benisty et al. 2018). Thus, this 'broken inner disc' scenario could explain dippers with a range of outer disc inclinations, including those with low or moderate outer disc inclinations, although it requires a stellar or massive planetary companion orbiting in the inner disc. Our AO imaging can rule out stellar (but not planetary) companions at a few au around some dippers in our sample (see Table A1). Although stellar companions in the inner $(<1 \mathrm{au})$ disc would not be resolved in our AO imaging, they could be detected as spectroscopic binaries. Moreover, even though many of the ALMA images presented here do not show inner gaps (the rate of these 'transition' discs in our sample is similar to that seen in the general disc population; see Ansdell et al. 2016c), the spatial resolutions - even of the DSHARP sample $(\sim 5 \mathrm{au})$ - are insufficient to resolve such small inner cavities.

It is of course possible that more than one (or all) of these mechanisms are responsible for the dipper phenomenon. Fig. 7 illustrates which mechanisms can be ruled out for the dippers in our sample based on the above discussion of disc geometry and currently available data.

\section{CONCLUSIONS}

Dippers are a common class of young variable star often assumed to host protoplanetary discs viewed nearly-edge on, such that dusty structures lifted slightly out of the mid-plane partially occult the star as the disc rotates, producing the characteristic dimming events seen in their optical light curves. Until recently, it was difficult to robustly test this assumption of disc geometry due to the limited number of dippers with resolved discs. This has changed with the advent of ALMA and recent flurry of protoplanetary disc observations conducted at moderate ( $\gtrsim 10 \mathrm{au}$ ) angular resolution (e.g. Barenfeld et al. 2016; Cieza et al. 2018).

Motivated by the earlier discovery of a dipper disc with a faceon geometry (J1604; Ansdell et al. 2016a), we investigated the distribution of dipper disc inclinations resolved by ALMA. We found a disc inclination distribution consistent with isotropic over $i_{\mathrm{d}} \approx 0-75^{\circ}$ (with a deficit at higher inclinations being consistent with an observational bias due to optically thick disc mid-planes 
blocking their host stars). We also found diverse disc morphologies on the $\gtrsim 10$ au scales typically probed by our ALMA observations, also evident at $\sim 5$ au scales for the four dippers observed at high spatial resolution as part of DSHARP (Andrews et al. 2018).

These findings indicate that the dipper phenomenon is unrelated to the outer ( $>10 \mathrm{au}$ ) disc geometry probed by ALMA, and that any connection with disc morphology remains unclear. Given several lines of evidence that the dipper events are caused by dust in the inner $(<1 \mathrm{au})$ disc, these findings further hint that inner disc misalignments may be common in protoplanetary discs around latertype stars. This interpretation is supported by recent results from high-contrast optical/infrared imagers that have revealed outer disc shadows likely cast by unseen misaligned inner disc components (e.g. Debes et al. 2017; Benisty et al. 2018; Casassus et al. 2018; Pinilla et al. 2018b). Such misaligned discs would be distinct from UXOR systems, which are expected to have aligned inner and outer disc components in order to explain both their dimming events (caused by a puffed-up inner disc rim) and their weak far-infrared emission (due to self-shadowing of the outer disc by the inner disc rim) (Dullemond et al. 2003). Potential mechanisms causing the inner disc features hypothesized for the dippers include accretiondriven inner disc warping and 'breaking' of the inner and outer disc due to (sub-)stellar or planetary companions on inclined orbits.

There are several important avenues for future work. Higher contrast optical/infrared observations from instruments like SPHERE and GPI are needed to search for fainter companions, in particular among the transition discs whose inner cavities may be carved out by planetary-mass objects. Moreover, multi-epoch observations from such instruments will give insight into the occurrence and variability of shadows in the outer disc, and thus the presence of unseen misaligned inner disc components. High-resolution spectra, probably obtained in the infrared where there is more signal from these late-type and reddened stars, could identify spectroscopic binaries that can 'break' circumbinary discs. Inclinations of the rotational axis of the central stars (derived from measurements of $v \sin i$, stellar rotation period, and stellar radius) can also be compared to the outer disc inclinations, as any difference would further indicate misalignments in discs (an initial attempt using literature values has been conducted by Davies 2019). Finally, given that the photometric behaviour and mid-infrared excesses of dippers are known to change on time-scales of months to years, simultaneous multiwavelength observations and long-term monitoring will be critical for better understanding this common class of young variable and thus the dynamic inner regions of protoplanetary discs more generally.

\section{ACKNOWLEDGEMENTS}

MA gratefully acknowledges the NVIDIA Corporation for donating the Quadro P6000 GPU used for this research. MA acknowledges support from National Science Foundation (NSF) grant AST1518332 and National Aeronautics and Space Administration (NASA) grants NNX15AC89G and NNH18ZDA001N/EW. MA, GD, and IA acknowledge support from NASA grant NNX15AD95G/NEXSS. MT is supported by the UK Science and Technology Research Council (STFC). JC acknowledges support from the NASA under grant No. 15XRP15 20140 issued through the Exoplanets Research programme. GMK is supported by the Royal Society as a Royal Society University Research Fellow. We thank Scott Barenfeld and Lucas Cieza for providing the calibrated ALMA visibilities of their disc survey programmes. Part of this research was carried out at the Jet
Propulsion Laboratory, Caltech, under a contract with NASA. This paper includes data from the Kepler mission; funding for Kepler is provided by the NASA Science Mission directorate. Some data used in this paper were obtained from the Mikulski Archive for Space Telescopes (MAST) at the Space Telescope Science Institute (STScI). STScI is operated by the Association of Universities for Research in Astronomy, Inc., under NASA contract NAS5-26555. We used data from the following ALMA programmes: 2011.0.00526.S, 2012.1.00046.S, 2013.1.00395.S, 2013.1.00498.S, 2015.1.01600.S, 2016.1.00336.S, 2016.1.00545.S, 2017.1.01180.S, and 2018.1.00564.S. ALMA is a partnership of ESO (representing its member states), NSF (USA) and NINS (Japan), together with NRC (Canada), NSC and ASIAA (Taiwan), and KASI (Republic of Korea), in cooperation with the Republic of Chile. The Joint ALMA Observatory is operated by ESO, AUI/NRAO, and NAOJ. The National Radio Astronomy Observatory (NRAO) is a facility of the NSF operated under cooperative agreement by Associated Universities, Inc. This work has made use of data from the European Space Agency (ESA) mission Gaia (https://www.cosmos.esa.int/gaia), processed by the Gaia Data Processing and Analysis Consortium (DPAC, https://www.cosmos.esa.int/web/gaia/dpac/consortium). Funding for the DPAC has been provided by national institutions, in particular the institutions participating in the Gaia Multilateral Agreement. Some of the data presented herein were obtained at the W. M. Keck Observatory, which is operated as a scientific partnership among the California Institute of Technology, the University of California and NASA. The Observatory was made possible by the generous financial support of the W. M. Keck Foundation. The authors wish to recognize and acknowledge the very significant cultural role and reverence that the summit of Maunakea has always had within the indigenous Hawaiian community. We are most fortunate to have the opportunity to conduct observations from this mountain. We used the following software: LIGHTKURVE (Lightkurve Collaboration 2018), ASTROPY (Astropy Collaboration 2013, 2018), MATPLOTLIB (Hunter 2007), UVPLOT (Tazzari 2017), and GALARIO (Tazzari et al. 2018).

\section{REFERENCES}

Alencar S. H. P. et al., 2010, A\&A, 519, A88 ALMA Partnership et al., 2015, ApJ, 808, L3

Andrews S. M. et al., 2016, ApJ, 820, L40

Andrews S. M. et al., 2018, ApJ, 869, L41

Ansdell M., Gaidos E., Williams J. P., Kennedy G., Wyatt M. C., LaCourse

D. M., Jacobs T. L., Mann A. W., 2016a, MNRAS, 462, L101

Ansdell M. et al., 2016b, ApJ, 816, 69

Ansdell M. et al., 2016c, ApJ, 828, 46

Astropy Collaboration, 2013, A\&A, 558, A33

Astropy Collaboration, 2018, AJ, 156, 123

Baglin A. et al., 2006, in COSPAR Meeting, 36th COSPAR Scientific Assembly, Beijing, China, p. 3749

Bans A., Königl A., 2012, ApJ, 758, 100

Barenfeld S. A., Carpenter J. M., Ricci L., Isella A., 2016, ApJ, 827, 142

Barenfeld S. A., Carpenter J. M., Sargent A. I., Isella A., Ricci L., 2017, ApJ, 851, 85

Barenfeld S. A. et al., 2019, ApJ, 878, 45

Benisty M. et al., 2018, A\&A, 619, A171

Beuzit J. L. et al., 2019, A\&A, 631, A155

Blandford R. D., Payne D. G., 1982, MNRAS, 199, 883

Bodman E. H. L. et al., 2017, MNRAS, 470, 202

Borucki W. J. et al., 2010, Science, 327, 977

Bouvier J. et al., 1999, A\&A, 349, 619

Bouvier J. et al., 2003, A\&A, 409, 169 
Bouvier J., Grankin K., Ellerbroek L. E., Bouy H., Barrado D., 2013, A\&A, 557, A77

Carpenter J. M., Ricci L., Isella A., 2014, ApJ, 787, 42

Casassus S. et al., 2018, MNRAS, 477, 5104

Cheetham A. C., Kraus A. L., Ireland M. J., Cieza L., Rizzuto A. C., Tuthill P. G., 2015, ApJ, 813, 83

Cieza L. A. et al., 2010, ApJ, 712, 925

Cieza L. A. et al., 2018, MNRAS, 474, 4347

Cody A. M., Hillenbrand L. A., 2018, AJ, 156, 71

Cody A. M. et al., 2014, AJ, 147, 82

Cox E. G. et al., 2017, ApJ, 851, 83

Davies C. L., 2019, MNRAS, 484, 1926

Davies C. L. et al., 2018, ApJ, 866, 23

Debes J. H. et al., 2017, ApJ, 835, 205

Draine B. T., Lee H. M., 1984, ApJ, 285, 89

Duchêne G., Kraus A., 2013, ARA\&A, 51, 269

Dullemond C. P., van den Ancker M. E., Acke B., van Boekel R., 2003, ApJ, 594, L47

Eisner J. A., Lane B. F., Hillenbrand L. A., Akeson R. L., Sargent A. I., 2004, ApJ, 613, 1049

Ellerbroek L. E. et al., 2014, A\&A, 563, A87

Erickson K. L., Wilking B. A., Meyer M. R., Robinson J. G., Stephenson L. N., 2011, AJ, 142, 140

Facchini S., Juhász A., Lodato G., 2018, MNRAS, 473, 4459

Fairlamb J. R., Oudmaijer R. D., Mendigutía I., Ilee J. D., van den Ancker M. E., 2015, MNRAS, 453, 976

Fazio G. G. et al., 2004, ApJS, 154, 10

Fernandes R. B. et al., 2018, ApJ, 856, 103

Foreman-Mackey D., Hogg D. W., Lang D., Goodman J., 2013, PASP, 125, 306

Gagné J. et al., 2018, ApJ, 856, 23

Giacalone S., Teitler S., Königl A., Krijt S., Ciesla F. J., 2019, ApJ, 882, 33

Grady C. A. et al., 2005, ApJ, 630, 958

Harris R. J., Andrews S. M., Wilner D. J., Kraus A. L., 2012, ApJ, 751, 115

Hedges C., Hodgkin S., Kennedy G., 2018, MNRAS, 476, 2968

Herbst W., Herbst D. K., Grossman E. J., Weinstein D., 1994, AJ, 108, 1906

Howell S. B. et al., 2014, PASP, 126, 398

Huang J. et al., 2018, ApJ, 869, L42

Hunter J. D., 2007, Comput. Sci. Eng., 9, 90

Ireland M. J., 2013, MNRAS, 433, 1718

Kesseli A. Y. et al., 2016, ApJ, 828, 42

Kraus A. L., Ireland M. J., Martinache F., Lloyd J. P., 2008, ApJ, 679, 762

Kraus A. L., Ireland M. J., Huber D., Mann A. W., Dupuy T. J., 2016, AJ, 152,8

Kreplin A., Madlener D., Chen L., Weigelt G., Kraus S., Grinin V., Tambovtseva L., Kishimoto M., 2016, A\&A, 590, A96

Kurosawa R., Romanova M. M., 2013, MNRAS, 431, 2673

Lightkurve Collaboration, 2018, Astrophysics Source Code Library, record ascl: 1812.013

Lin M.-K., 2013, ApJ, 765, 84

Lindegren L. et al., 2018, A\&A, 616, A2

Long F. et al., 2019, ApJ, 882, 49

Loomis R. A., Öberg K. I., Andrews S. M., MacGregor M. A., 2017, ApJ, 840,23

Lovelace R. V. E., Li H., Colgate S. A., Nelson A. F., 1999, ApJ, 513, 805

Luger R., Agol E., Kruse E., Barnes R., Becker A., Foreman-Mackey D., Deming D., 2016, AJ, 152, 100

Luhman K. L., Mamajek E. E., 2012, ApJ, 758, 31

Lyra W., Mac Low M.-M., 2012, ApJ, 756, 62

McClure M. K. et al., 2010, ApJS, 188, 75

McGinnis P. T. et al., 2015, A\&A, 577, A11

Manara C. F., Testi L., Natta A., Alcalá J. M., 2015, A\&A, 579, A66

Marino S., Perez S., Casassus S., 2015, ApJ, 798, L44

Martinez A. O. et al., 2017, ApJ, 837, 72
Mathews G. S., Williams J. P., Ménard F., 2012, ApJ, 753, 59

Matsakos T., Königl A., 2017, AJ, 153, 60

Mayama S. et al., 2018, ApJ, 868, L3

Meheut H., Yu C., Lai D., 2012a, MNRAS, 422, 2399

Meheut H., Keppens R., Casse F., Benz W., 2012b, A\&A, 542, A9

Meheut H., Lovelace R. V. E., Lai D., 2013, MNRAS, 430, 1988

Ménard F., Bouvier J., Dougados C., Mel'nikov S. Y., Grankin K. N., 2003, A\&A, 409, 163

Min M., Stolker T., Dominik C., Benisty M., 2017, A\&A, 604, L10

Miranda R., Lai D., Méheut H., 2016, MNRAS, 457, 1944

Miranda R., Li H., Li S., Jin S., 2017, ApJ, 835, 118

Morales-Calderón M. et al., 2011, ApJ, 733, 50

Nealon R., Pinte C., Alexander R., Mentiplay D., Dipierro G., 2019, MNRAS, 484, 4951

Ono T., Muto T., Takeuchi T., Nomura H., 2016, ApJ, 823, 84

Pecaut M. J., Mamajek E. E., 2016, MNRAS, 461, 794

Pinilla P. et al., 2018a, ApJ, 859, 32

Pinilla P. et al., 2018b, ApJ, 868, 85

Pinte C., Ménard F., Duchêne G., Bastien P., 2006, A\&A, 459, 797

Preibisch T., Brown A. G. A., Bridges T., Guenther E., Zinnecker H., 2002, AJ, 124, 404

Ratzka T., Köhler R., Leinert C., 2005, A\&A, 437, 611

Rich E. A. et al., 2015, AJ, 150, 86

Ricker G. R. et al., 2014, in Oschmann J. M. Jr, Clampin M., Fazio G. G., MacEwen H. A., eds, Proc. SPIE Conf. Ser. Vol. 9143, Space Telescopes and Instrumentation 2014: Optical, Infrared, and Millimeter Wave. SPIE, Bellingham, p. 914320

Rizzuto A. C., Ireland M. J., Kraus A. L., 2015, MNRAS, 448, 2737

Schneider P. C., Manara C. F., Facchini S., Günther H. M., Herczeg G. J., Fedele D., Teixeira P. S., 2018, A\&A, 614, A108

Stauffer J. et al., 2017, AJ, 153, 152

Stolker T. et al., 2016, A\&A, 595, A113

Takami M. et al., 2014, ApJ, 795, 71

Tambovtseva L. V., Grinin V. P., 2008, Astron. Lett., 34, 231

Tazzari M., 2017, mtazzari/uvplot: v0.1.1, https://doi.org/10.5281/zenodo.1 003113

Tazzari M., Beaujean F., Testi L., 2018, MNRAS, 476, 4527

Teague R., Bae J., Bergin E. A., Birnstiel T., Foreman-Mackey D., 2018, ApJ, 860, L12

van Dam M. A., Le Mignant D., Macintosh B. A., 2004, Appl. Opt., 43, 5458

van Dam M. A. et al., 2006, PASP, 118, 310

van der Plas G. et al., 2016, ApJ, 819, 102

Vanderburg A., Johnson J. A., 2014, PASP, 126, 948

Varga J. et al., 2017, A\&A, 604, A84

Wilking B. A., Meyer M. R., Robinson J. G., Greene T. P., 2005, AJ, 130, 1733

Williams J. P., Cieza L., Hales A., Ansdell M., Ruiz-Rodriguez D., Casassus S., Perez S., Zurlo A., 2019, ApJ, 875, L9

Wizinowich P. et al., 2000, PASP, 112, 315

Wizinowich P. L. et al., 2006, PASP, 118, 297

Wright E. L. et al., 2010, AJ, 140, 1868

Yelda S., Lu J. R., Ghez A. M., Clarkson W., Anderson J., Do T., Matthews K., 2010, ApJ, 725, 331

Zhang K., Isella A., Carpenter J. M., Blake G. A., 2014, ApJ, 791, 42

Zhu Z., 2019, MNRAS, 483, 4221

Ziegler C., Tokovinin A., Briceno C., Mang J., Law N., Mann A. W., 2019, preprint (arXiv:1908.10871)

\section{APPENDIX A: IMAGING DETECTION LIMITS}

Table A1 presents the detection limits derived from high-contrast imaging of the dippers in our sample, when available. The derivation of the contrast limits for the sources observed as part of this work are described in Section 2.4 and we provide references for those values taken from the literature. Some sources have more than one entry when different works probed different separations. 


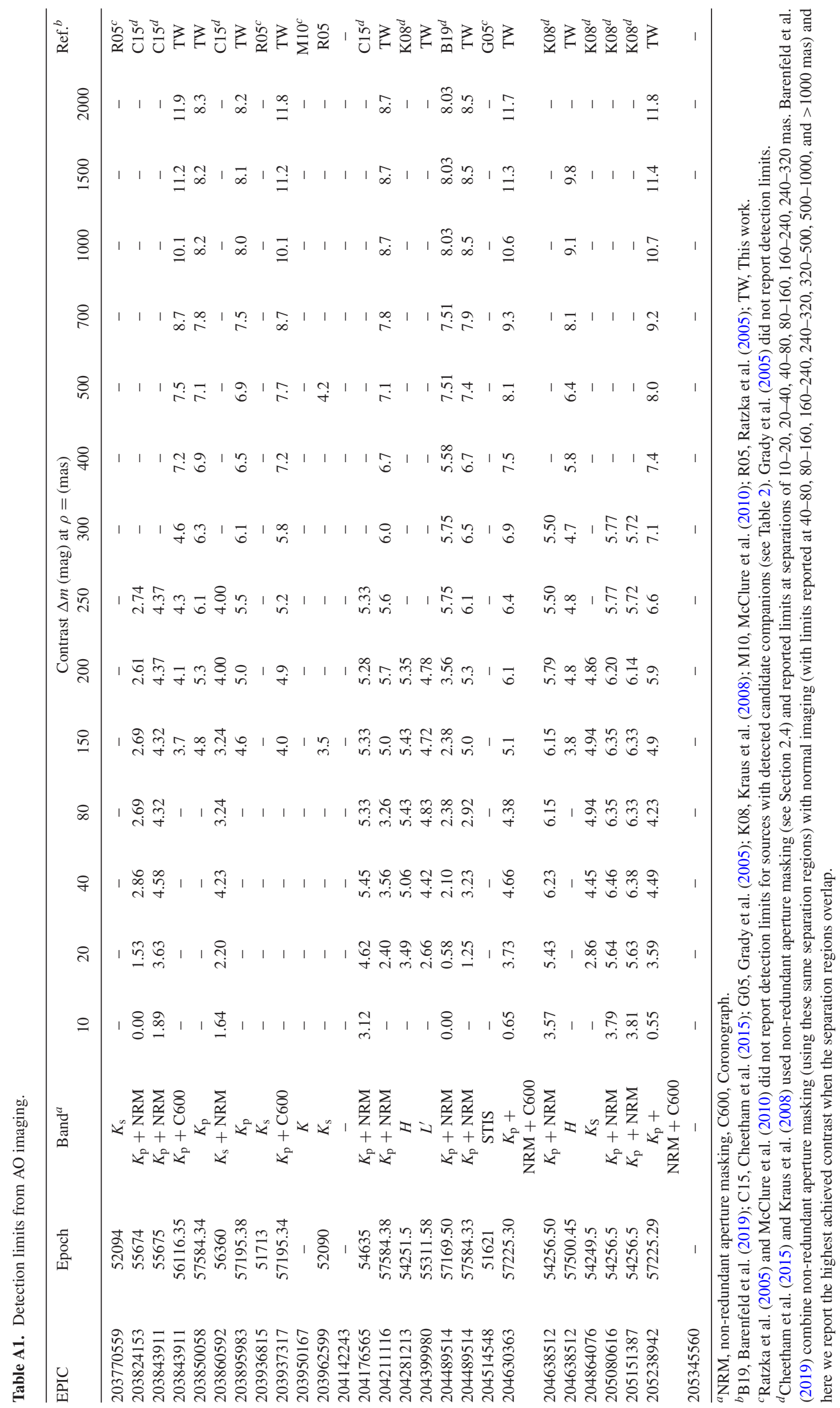




\section{APPENDIX B: MCFOST MODEL GRID}

Our MCFOST models (Pinte et al. 2006) all assume axisymmetric, tapered-edge disc profiles that are one of the standard options of the software package. We generate the model grid by varying six parameters: dust mass $\left(M_{\text {dust }}=1 \times 10^{-6}, 1 \times 10^{-5}, 1 \times 10^{-4}\right.$, $\left.1 \times 10^{-3} \mathrm{M}_{\odot}\right)$, critical radius $\left(R_{\mathrm{c}}=10,30,100 \mathrm{au}\right)$, flaring exponent $\left(f_{\exp }=1.0,1.15,1.30\right)$, scale height $\left(H_{0}=5,10,15,20\right.$ au at 100 au radius), surface density exponent $(\gamma=0.0,0.5,1.0,1.5)$, and maximum grain size $\left(a_{\max }=10,100,1000,10000 \mu \mathrm{m}\right)$. To better represent empirical distributions of these model parameters, we weight $M_{\text {dust }}$ according to the distribution in Taurus, and weight $H_{0}$ based on the distribution of mid-plane temperatures across the entire grid, which drastically reduces the $H_{0}=20$ au models as few discs are this hot. We also fix the stellar effective temperature $\left(T_{\text {eff }}=4000 \mathrm{~K}\right)$, inner radius $\left(R_{\mathrm{in}}=0.1 \mathrm{au}\right)$, and minimum grain size $\left(a_{\min }=0.01 \mu \mathrm{m}\right)$, while assuming Mie scattering and astronomical silicates (similar to Draine \& Lee 1984) for the dust grain properties.

For each combination of model parameters, we generate a spectral energy distribution (SED) at 15 different inclinations from $i_{\mathrm{d}}=$ $45-90^{\circ}$ spaced uniformly in $\cos \left(i_{\mathrm{d}}\right)$, then compute the fraction of models at a given inclination for which the flux from the system is at least 40 times weaker than that of the host star. This factor of 40 is chosen based on the median Kepler magnitude of our sample of $K_{\mathrm{p}} \approx 13 \mathrm{mag}$ and the Kepler faint limit of $K_{\mathrm{p}} \approx 17 \mathrm{mag}$. We find that $\gtrsim 50$ percent of the model grid would be undetectable by Kepler for discs with $i_{\mathrm{d}} \gtrsim 80^{\circ}$, a function that rises steeply as $\gtrsim 90$ per cent would be undetectable for $i_{\mathrm{d}} \gtrsim 85^{\circ}$.

\section{APPENDIX C: REJECTED DIPPERS WITH INCLINATIONS REPORTED IN LITERATURE}

Several dippers in Upper Sco have disc inclinations derived from ALMA data reported in the literature but are not included in our sample due to their large uncertainties. Indeed, the visibilities of all of these sources are flat or nearly flat with UV distance (see fig. 1 in Barenfeld et al. 2017), indicating they are unresolved or poorly resolved. This make them distinct from the sources in our sample,

Table C1. Rejected dippers with inclinations reported in literature.

\begin{tabular}{lccccc}
\hline EPIC & SpT. & Ref. SpT & $i_{\mathrm{d}, \text { lit }}$ & Ref. $i_{\mathrm{d}, \text { lit }}$ & $i_{\mathrm{d}}$ \\
\hline 203750883 & M3.5 & L12 & $86_{-52}^{+4}$ & B17 & - \\
204245509 & K2 & L12 & $4_{-3}^{+48}$ & B17 & - \\
204278916 & M1 & L12 & $57_{-14}^{+14}$ & B17 & $51_{-17}^{+14}$ \\
204757338 & M4.5 & A16 & $68_{-49}^{+10}$ & B17 & $56_{-27}^{+18}$ \\
204932990 & M3.5 & L12 & $86_{-42}^{+4}$ & B17 & - \\
205037578 & M3.5 & L12 & $80_{-50}^{+7}$ & B17 & - \\
205241182 & M4.5 & L12 & $71_{-63}^{+8}$ & B17 & - \\
205383125 & M3 & L12 & $86_{-60}^{+4}$ & B17 & - \\
\hline
\end{tabular}

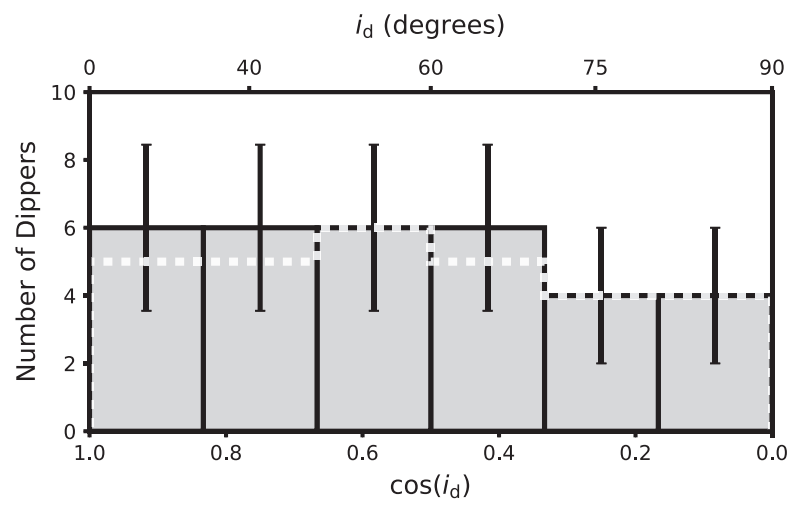

Figure C1. The inclination distribution of dipper discs resolved by ALMA, now including those with large uncertainties from the literature that were used in Cody \& Hillenbrand (2018) to suggest a bias towards highly inclined discs amongst the dippers. The disc inclination values were taken from Tables 1 and C1, and the symbols are the same as in Fig. 3.

which all have clearly declining visibilities with UV distance (see Fig. 1). The excluded sources are given in Table $\mathrm{C} 1$, which has the same column header meanings and references as Table 1. For most of these sources we cannot derive any inclination using our method (Section 3.1), however for two discs we could extract inclinations consistent with those in the literature, albeit with large $\left(\gtrsim 20^{\circ}\right)$ uncertainties (using the archival data from 2013.1.00395.S for EPIC 204278916 and data from our targeted ALMA programme 2016.1.00336.S for EPIC 204757338) as given in Table C1. A handful of $\rho$ Oph dippers also have marginally resolved discs with large $\left(\gtrsim 20^{\circ}\right)$ inclination uncertainties when fit with our method; these are also not included in our sample.

We do not include these marginally resolved sources in our sample because the key algorithm implemented in EMCEE has difficulties handling multimodal posterior distributions, such as those of the inclination parameter due to its symmetry (i.e. $i_{\mathrm{d}}=$ $\pm 20^{\circ}$ are equally reasonable solutions), when the source is only marginally resolved and/or the signal-to-noise ratio is low. This is because the walker-based algorithm will still explore both posterior peaks, even if the priors are constrained to one period (i.e. $i_{\mathrm{d}}=$ $\left.\left[0^{\circ}, 90^{\circ}\right]\right)$, causing tails in the distribution that are artefacts of the algorithm rather than reliable posterior samples.

Nevertheless, including the sources in Table $\mathrm{C} 1$ with the inclinations reported in the literature would not change our overall results. In fact, if taken at face value, they would actually make the distribution even more consistent with isotropic by filling out the higher inclinations, as shown in the histogram in Fig. C1. We do not construct an ECDF as in Section 4.1 because the MCMC posterior distributions are unreliable, for the reasons described above.

This paper has been typeset from a $\mathrm{T}_{\mathrm{E}} \mathrm{X} / \mathrm{LT}_{\mathrm{E}} \mathrm{X}$ file prepared by the author. 